\title{
Linear stability of confined flow around a 180-degree sharp bend
}

\author{
Azan M. Sapardi ${ }^{1,2}$, Wisam K. Hussam ${ }^{1,3}$, Alban Pothérat $^{4}$ \\ and Gregory J. Sheard ${ }^{1}$ \\ ${ }^{1}$ The Sheard Lab, Department of Mechanical and Aerospace Engineering, \\ Monash University, Victoria 3800, Australia \\ Greg.Sheard@monash.edu \\ ${ }^{2}$ Department of Mechanical Engineering, International Islamic University Malaysia, \\ Kuala Lumpur 53300, Malaysia \\ ${ }^{3}$ School of Engineering, Australian College of Kuwait, \\ Safat 13015, Kuwait \\ ${ }^{4}$ Applied Mathematics Research Centre, \\ Coventry University, Coventry CV1 5FB, United Kingdom
}

November 15, 2018

\begin{abstract}
This study seeks to characterise the breakdown of the steady two-dimensionalsolution in the flow around a 180-degree sharp bend to infinitesimal three-dimensional disturbances using a linear stability analysis. The stability analysis predicts that three-dimensional transition is via a synchronous instability of the steady flows. A highly accurate global linear stability analysis of the flow was conducted with Reynolds number $R e<1150$ and bend opening ratio (ratio of bend width to inlet height) $0.2 \leq \beta \leq 5$. This range of $R e$ and $\beta$ captures both steadystate two-dimensional flow solutions as well as the inception of unsteady two-dimensional flow. For $0.2 \leq \beta \leq 1$, the two-dimensional base flow transitions from steady to unsteady at higher Reynolds number as $\beta$ increases. The stability analysis shows that at the onset of instability, the base flow becomes three-dimensionally unstable in two different modes, namely spanwise oscillating mode for $\beta=0.2$, and spanwise synchronous mode for $\beta \geq 0.3$. The critical Reynolds number and the spanwise wavelength of perturbations increase as $\beta$ increases. For $1<\beta \leq 2$ both the critical Reynolds for onset of unsteadiness and the spanwise wavelength decrease as $\beta$ increases. Finally, for $2<\beta \leq 5$, the critical Reynolds number and spanwise wavelength remain almost constant. The linear stability analysis also shows that the base flow becomes unstable to different three-dimensional modes depending on the opening ratio. The modes are found to be localised near the reattachment point of the first recirculation bubble.
\end{abstract}

\section{Introduction}

An important geometric feature of the ductwork carrying liquid-metal coolant fluid within prototype blankets in magnetic confinement fusion reactors is the presence of sharp 180-degree bends (Boccaccini et al. 2004, Kirillov et al. 1995, Barleon et al., 1991, 1996, Bühler, 2007). The transport of heat from the far side-wall of the bend and the flow around the bend are critical aspects for the efficient transport of heat from the reactor for power generation (Boccaccini et al. 2004$)$. 
Despite its relative simplicity, few studies have been conducted on the hydrodynamic flow in this geometry, and they have focused mostly on heat transfer. An understanding of this flow underpins the duct flow problem with application to fusion reactor blankets.

Fundamentally, the two-dimensional flow around the sharp bend creates recirculation structures that resemble those seen in several canonical confined flow problems, including the backward-facing step flow (Armaly et al., 1983, Ghia et al., 1989, Barkley et al., 2002, Blackburn et al., 2008). These flows exhibit a streamline emerging from the upstream separation point that divides regions of reversed flow from the main bulk flow, and is termed the dividing streamline. When the dividing streamline reattaches to the wall downstream, a closed separation bubble is formed. Specifically, the flow first passes over a large recirculation bubble attached to the downstream side of the inner corner of the bend, and under same conditions, subsequently a second recirculation bubble develops on the opposite wall a little further downstream. Separating and reattaching flows play an important role in numerous engineering applications, especially in heat transportation (Krall \& Sparrow, 1966, Abu-Nada, 2008; Larson, 1959).

There have been a number of studies on the effect of different geometric parameters on the efficiency of heat transfer in flows around a bend. The effects of a duct with a 180-degree bend with three different turning configurations: sharp corner, rounded corner and circular turn were studied by Wang \& Chyu (1994). In their study, all walls were heated and cold fluid was supplied from the inlet. Their results show that the sharp bend had the strongest turn-induced heat transfer enhancement by approximately $30 \%$ as compared to the circular turn which has the weakest among those three configurations. Heat transfer was found to be optimum downstream of the sharp bend turn (experimental studies by Metzger \& Sahm (1986) and Astarita \& Cardone (2000)). In an experimental study on the effect of the size of bend openings in ducts with sharp bend by Hirota et al. (1999), with a channel cross-section of $50 \times 25 \mathrm{~mm}$, bend openings of $70 \mathrm{~mm}$ and $50 \mathrm{~mm}$ were found to have almost the same value of Sherwood number $S h$ (the ratio of convective to diffusive mass transport), whereas a smaller bend opening $(30 \mathrm{~mm})$ led to values of $S h 1.3$ times larger than that for $50 \mathrm{~mm}$. Only a few studies focused on the effect of controllable parameters on the structure of the flow. Liou et al. $(1999,2000)$ experimentally showed that the divider thickness (the thickness of the structure between the inflow and outflow channels) mainly influenced the intensity and uniformity of turbulence.

The only reported parametric study on the effect of the bend opening ratio on hydrodynamic flow around a 180-degree sharp bend was carried out numerically by Zhang \& Pothérat (2013). They categorised the two-dimensional flow into five regimes (see figure 4 in Zhang \& Pothérat, 2013). Regime I was at very low Re, where the flow was laminar and remained attached to the walls throughout. Regime II emerged at higher Re, where flow separation appears at the bend, leading to the creation of the primary recirculation bubble. Regime III occurred at a yet higher Reynolds number when the adverse pressure gradient at the top wall was strong enough to create a secondary recirculation bubble there. Regime IV occurred when a small scale vortex structure was detected far downstream at high $R e$ before the flow entered regime $\mathrm{V}$, which featured vortex shedding originating from the sharp bend at higher Re. They also reported the results of some three-dimensional simulations. According to their results, at $R e=2000$, two types of shedding structures were found in the spanwise direction reminiscent of A- and B-modes in the flow past a circular cylinder (Williamson, 1988: Thompson et al., 1996: Brede et al. 1996; Henderson, 1997). These shedding structures disrupted the two-dimensional vortex in the flow and tended to slow down the shedding mechanism. Though a very detailed study of the two-dimensional 180-degree bend flow has been provided by Zhang \& Pothérat (2013), the three-dimensional stability of these flows is yet to be determined.

One approach towards understanding the three-dimensional stability relies on a linear stability analysis, where the stability of infinitesimal three-dimensional perturbations to a two-dimensional 
flow is determined by obtaining the leading eigenmode(s) of the evolution operator of the linearised perturbation field. Combined with accurate numerical methods, this technique has been significantly contributing to a better understanding of separated flows in complex geometries over the past couple of decades. Relevant examples include backward-facing step (Barkley et al. 2002 , Blackburn et al. 2008) and partially blocked channel flows (Griffith et al. 2008).

Studies elucidating the stability and the three-dimensionality of the flow past a backward-facing step include Armaly et al. (1983); Ghia et al. (1989); Barkley et al. (2002); Wee et al. (2004); Griffith et al. (2007) and Lanzerstorfer \& Kuhlmann (2012). Based on detailed experiments, Ghia et al. (1989) and Armaly et al. (1983) initially proposed that the curvature in the main flow caused by the second recirculation bubble led to a Taylor-Görtler instability. This type of instability occurs in flows with curved streamlines when the fluid velocity decreases radially, and the centrifugal force drives pairs of counter-rotating streamwise vortices (Drazin \& Reid, 2004). However, the linear stability analysis of Barkley et al. (2002) ruled out the effect of a Taylor-Görtler-type instability because they found that the two-dimensional flow remained linearly stable long after the secondary recirculation bubble appeared. Instead, they found the critical eigenmode to consist of a flat roll localised in the primary recirculation region at the step edge. Hammond \& Redekopp (1998) studied the instability properties of separation bubbles. They found that the instability mode associated to the inflection point of the dividing streamline became globally unstable as the peak backflow velocity approached about $30 \%$ of the free stream value. As the size of the bubble grew, the peak velocity of the backflow increased correspondingly and the conditions for local absolute instability could be predicted.

No such scenario has been established for the onset of unsteadiness of flows in 180-degree sharp bends. The purpose of the present paper is to find such a scenario using methods in the spirit of Barkley et al. (2002). The specific aim is to thoroughly characterise the three-dimensional stability of flow around a 180-degree sharp bend as a function of Reynolds number, bend opening ratio, and spanwise wavenumber of the three-dimensional disturbances. In turn it is expected that this study will provide insights into a more general separated confined flows, and this understanding may enable future enhancement of the efficiency of heat transport in such systems.

This paper is organised as follows. Problem formulation and numerical methods are presented in $\S 2$ and $\S 3$, respectively. In $\S 4$ and $\S 5$, respectively, the base flow characteristics and the results of the stability analysis for a range of $\beta$ and $R e$ are discussed. Finally, the nature of bifurcation in the three-dimensional flow is studied in $\S 6$.

\section{Problem formulation}

Figure 1 shows the computational domain under consideration, including the geometric parameters for the problem. The channel widths in the inlet and at the bend are $a$ and $b$, respectively. The heights of the inlet and outlet channels are identical. The divider thickness is $c$, with $d$ and $e$ respectively denoting the lengths from the far wall of the bend to the inlet and outlet, respectively. The ratio of the gap $c$ to the channel height $a$ is $4 \%$, while the lengths of the upstream and downstream channels are $(d-b)=15 a$ and $(e-b)=30 a$. The opening ratio of the bend is defined as $\beta=b / a$.

The fluid has a constant density $\rho$ and kinematic viscosity $\nu$. In this study, velocities are normalised by the peak inlet velocity $U_{o}$, lengths by inlet channel height $a$, time by $a / U_{o}$ and pressure by $\rho U_{o}^{2}$. The fluid motion is governed by the incompressible Navier-Stokes equations,

$$
\begin{gathered}
\frac{\partial \boldsymbol{u}}{\partial t}=\mathbf{N}(\boldsymbol{u})-\boldsymbol{\nabla} p+\frac{1}{R e} \nabla^{2} \boldsymbol{u}, \\
\boldsymbol{\nabla} \cdot \boldsymbol{u}=0,
\end{gathered}
$$




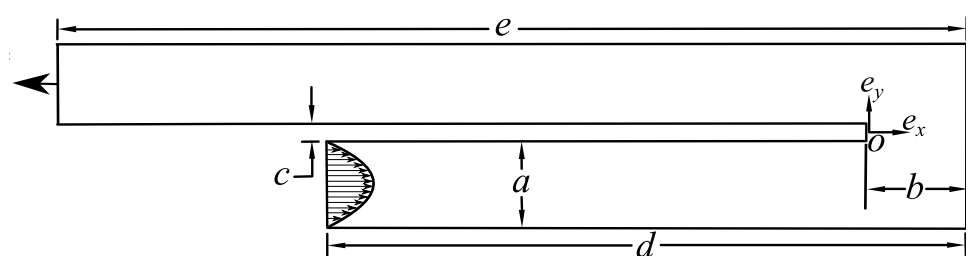

Figure 1: Flow geometry for the 180-degree sharp bend system. The fluid enters the bottom channel flowing to the right, and exits the top channel flowing leftwards.

where $\boldsymbol{u}$ is the velocity field, $p$ is the pressure, the Reynolds number

$$
R e \equiv \frac{U_{o} a}{\nu},
$$

and the non-linear advection term is calculated in convective form as $\mathbf{N}(\boldsymbol{u}) \equiv-(\boldsymbol{u} \cdot \boldsymbol{\nabla}) \boldsymbol{u}$.

Fluid enters from the inlet, flows around the sharp bend and through the outlet channel. With the origin of a Cartesian coordinate system positioned at the mid-point of the divider surface at the inside of the bend (see figure 1), boundary conditions are imposed as follows: at the inlet $(x=b-d,-1.02 \leq y \leq-0.02)$, a Poiseuille velocity profile $u_{x}=1-4(y+0.52)^{2}, u_{y}=0, u_{z}=0$ is imposed. A no slip boundary condition $(\boldsymbol{u}=0)$ is imposed at all solid walls. At the outlet $(x=b-e, 0.02 \leq y \leq 1.02)$, a standard outflow boundary is enforced with a Dirichlet reference pressure $(p=0)$ and a weakly enforced zero normal velocity gradient (Barkley et al., 2008).

\section{Computational methods}

The governing equations are spatially discretised using a spectral-element method and time integrated using a third-order backward differentiation scheme (Karniadakis et al., 1991). The twodimensional Cartesian formulation of the present code has been validated and employed in several confined channel-flow problems (e.g. Neild et al., 2010, Hussam et al., 2012a b). The method used to analyse the linear stability of perturbations is based on time integration of the linearized Navier-Stokes equations following Barkley \& Henderson (1996) and references therein. The technique is in fact a Floquet problem for time-periodic base flows, but is applied to the steady-state base flows in the present problem for convenience, as it was already implemented and validated within our code.

Velocity and pressure fields are decomposed into a two-dimensional base flow and infinitesimal fluctuating disturbance components

$$
\begin{gathered}
\boldsymbol{u}=\boldsymbol{u}_{2 \mathrm{D}}+\boldsymbol{u}^{\prime}, \\
p=p_{2 \mathrm{D}}+p^{\prime} .
\end{gathered}
$$

Substituting equations (4) and (5) into (1, 2) and retaining terms in the first order of the perturbation field yields the linearised Navier-Stokes equations describing the evolution of infinitesimal three-dimensional disturbances,

$$
\begin{gathered}
\frac{\partial \boldsymbol{u}^{\prime}}{\partial t}=-\mathbf{D N}\left(\boldsymbol{u}^{\prime}\right)-\nabla p^{\prime}+\frac{1}{R e} \nabla^{2} \boldsymbol{u}^{\prime}, \\
\boldsymbol{\nabla} \cdot \boldsymbol{u}^{\prime}=0,
\end{gathered}
$$

where we calculate the linearised advection term as $\mathbf{D N}\left(\boldsymbol{u}^{\prime}\right)=\left(\boldsymbol{u}_{2 \mathrm{D}} \cdot \boldsymbol{\nabla}\right) \boldsymbol{u}^{\prime}+\left(\boldsymbol{u}^{\prime} \cdot \boldsymbol{\nabla}\right) \boldsymbol{u}_{2 \mathrm{D}}$. 
Since the base flow is invariant in the spanwise direction, we can decompose general perturbations into Fourier modes with spanwise wavenumber

$$
k=\frac{2 \pi}{\lambda}
$$

where $\lambda$ is the wavelength in the spanwise direction. As per equation (6, 7), the equations are linear in $\boldsymbol{u}^{\prime}$ and therefore Fourier modes are linearly independent and coupled only with the twodimensional base flow. The absence of any spanwise component to the base flow further permits a single phase of the complex Fourier mode to be considered, i.e.

$$
\left.\begin{array}{rl}
\boldsymbol{u}^{\prime}(x, y, z, t) & =\langle\hat{u}(x, y, t) \cos (k z), \hat{v}(x, y, t) \cos (k z), \hat{w}(x, y, t) \sin (k z)\rangle \\
p^{\prime}(x, y, z, t) & =\hat{p}(x, y, t) \cos (k z)
\end{array}\right\} .
$$

The flow stability therefore reduces to a three-parameter problem in $R e, \beta$ and $k$. Following Barkley $\&$ Henderson (1996) and others, spanwise phase locked perturbations of the form in equation (9) remain in this form under the linearised evolution equations (7)-(6): for a $R e, \beta$ and spanwise wavenumber $k$, the three-dimensional/three-component perturbation field $\boldsymbol{u}^{\prime}(x, y, z, t)$ then reduces to a two-dimensional/three-component field

$$
\hat{\boldsymbol{u}}(x, y, t)=\langle\hat{u}(x, y, t), \hat{v}(x, y, t), \hat{w}(x, y, t)\rangle,
$$

which is computed on the same two-dimensional domain as the base flow. The perturbation $z$ velocity features a sine function rather than a cosine as only $z$-derivatives of this term (yielding a cosine) interact with other terms under (7)-(6).

By defining $\mathscr{A}(\tau)$ to represent the linear evolution operator for time integration (via equations (6) and (7)) of a perturbation field comprising a single phase-locked spanwise Fourier mode $\hat{\boldsymbol{u}}$ over time interval $\tau$, i.e.

$$
\hat{\boldsymbol{u}}(t+\tau)=\mathscr{A}(\tau) \hat{\boldsymbol{u}},
$$

an eigenvalue problem may then be constructed as

$$
\mathscr{A}(\tau) \hat{\boldsymbol{u}}_{k}=\mu_{k} \hat{\boldsymbol{u}}_{k},
$$

having complex eigenvalues $\mu_{k}$ and eigenvectors $\hat{\boldsymbol{u}}_{k}$. Eigenvalues $\mu_{k}$ are Floquet multipliers that relate to the eigenmode's exponential growth rate $\sigma$ and angular frequency $\omega$ through

$$
\mu \equiv e^{(\sigma+\mathrm{i} \omega) \tau},
$$

where the subscripts have been omitted for clarity. As the base flows are time-invariant in this study, the usual time period is replaced by an arbitrary time interval for $\tau$. An appealing feature of this technique is that solutions to the eigenvalue problem (12) may be obtained using iterative methods involving time-integration of the linearised perturbation field via (6)-(7), which avoids the substantial cost of explicitly constructing the very large operator $\mathscr{A}(\tau)$.

Stability is dictated by the leading eigenmode (i.e. $\mu=\mu_{k}$ having largest $\left.\left|\mu_{k}\right|\right)$. Neutral stability corresponds to $|\mu|=1$, while $|\mu|>1$ and $|\mu|<1$ describe unstable and stable flows, respectively. The bifurcation may be either synchronous $(\omega=0)$ or oscillatory $(\omega \neq 0)$. The smallest Reynolds number for which any spanwise wavenumber $k$ yields $|\mu|=1$ is the critical Reynolds number for the onset of instability.

The following steps are taken in order to solve this problem numerically. The time invariant base flow at a given $R e$ and $\beta$ is obtained by solving the two-dimensional Navier-Stokes equations (11)-(2) and stored. Subsequently, random initial perturbation fields are constructed for one or more spanwise wavenumbers $k$, and an implicitly restarted Arnoldi method in conjunction with time integration of the linearised Navier-Stokes equations (6)- $(77)$ is used to determine the leading eigenmodes governing stability. The ARPACK (Lehoucq et al., 1998) implementation of the implicitly restarted Arnoldi method is used, and the present formulation has been validated and employed across Sheard et al. (2009); Sheard (2011); Vo et al. (2014, 2015). 


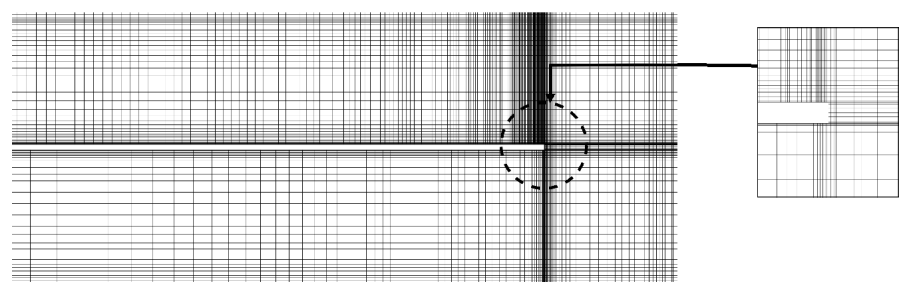

Figure 2: Details of the mesh around turning part area with polynomial order $N=3$.

$\begin{array}{ccccccccc}N & \mathrm{~A} & \mathrm{~B} & \mathrm{C} & \mathrm{D} & L_{\mathrm{R}_{1}} & L_{\mathrm{R}_{2}} & \% L_{\mathrm{R}_{1}} & \% L_{\mathrm{R}_{2}} \\ 4 & 0 & -4.8057 & -3.7077 & -9.7726 & 4.80573 & 6.06489 & 0.0307 & 0.0797 \\ 5 & 0 & -4.8072 & -3.7069 & -9.7765 & 4.80716 & 6.06955 & 0.0009 & 0.0029 \\ 6 & 0 & -4.8073 & -3.7071 & -9.7766 & 4.80730 & 6.06962 & 0.0019 & 0.0018 \\ 7 & 0 & -4.8073 & -3.7070 & -9.7766 & 4.80725 & 6.06966 & 0.0009 & 0.0010 \\ 8 & 0 & -4.8072 & -3.7069 & -9.7767 & 4.80720 & 6.06972 & - & -\end{array}$

Table 1: Dependence of recirculation length on polynomial order. Parameter $N$ indicates the independent polynomial order of the base flow. Two separation points (A and $\mathrm{C}$ ) and two reattachment points (B and $\mathrm{D}$ ) as indicated in figure 3 computed on the mesh at $R e=500$ and $\beta=1$ are given. $L_{\mathrm{R}_{1}}$ and $L_{\mathrm{R}_{2}}$ represent the recirculation length of the first and the second bubble, respectively. Errors on bubble lengths at each $N$ relative to the highest $N$ are also provided.

\subsection{Test of base flow structure computations}

The flow past a 180-degree sharp bend is a deceptively difficult problem to fully resolve, especially at large Reynolds number due to the sensitivity of the solution to the mesh structure mainly near the sharp bend. This section describes the tests used to validate the numerical algorithm, and to select appropriate meshes and element order. For the spatial resolution study, we varied element polynomial degree from $N=4$ to $N=8$ of a mesh based on domain length parameters from the mesh domain. For consistency with the domain size study, the mesh employed in this study models a 180-degree sharp bend with opening ratio $\beta=1$ and $R e=500$. In this regime, the flow is steady, with two recirculation bubbles. Figure 2 shows the detail of the mesh with $N=3$. The mesh is structured and refined in the vicinity of the sharp bend as well as in the downstream channel in order to capture the detailed structure of the flow that passes around the bend.

To demonstrate the accuracy of computing recirculation length in the base flow, table 1 shows the relative error of several measured quantities as a function of polynomial order. From computation of error on the recirculation length (Table 1), it was found that the polynomial order

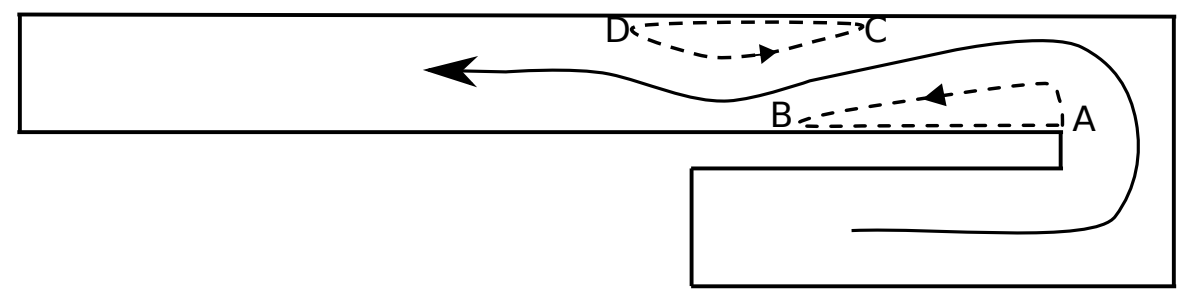

Figure 3: Sketch of separation and re-attachment points defining the locations of all recirculations. The unbroken arrow represents the direction of the main bulk flow as it navigates the bend. 


$\begin{array}{ccccc}\text { Outlet length }(e- & L_{\mathrm{R}_{1}} & L_{\mathrm{R}_{2}} & \% L_{\mathrm{R}_{1}} & \% L_{\mathrm{R}_{2}} \\ b) & & & & \\ 10 & 4.80485494 & 5.79491686 & 0.00723577 & 4.482661 \\ 20 & 4.80452028 & 6.06684499 & 0.00027037 & 0.000483 \\ 30 & 4.80452020 & 6.06685749 & 0.00026858 & 0.000277 \\ 40 & 4.80452009 & 6.06685810 & 0.00026644 & 0.000267 \\ 50 & 4.80451921 & 6.06685923 & 0.00024807 & 0.000248 \\ 60 & 4.80451788 & 6.06686092 & 0.00022029 & 0.000220 \\ 70 & 4.80451875 & 6.06685982 & 0.00023848 & 0.000239 \\ 80 & 4.80451505 & 6.06686448 & 0.00016141 & 0.000161 \\ 90 & 4.80450991 & 6.06687100 & 0.00005442 & 0.000054 \\ 100 & 4.80450729 & 6.06687431 & - & -\end{array}$

Table 2: Dependence of the length of the primary and secondary recirculation bubbles $\left(L_{\mathrm{R}_{1}}\right.$ and $L_{\mathrm{R}_{2}}$, respectively) on outlet channel length $(e-b)$. Percent differences between bubble lengths at each $e-b$ relative to the longest-outlet case $e-b=100$ are also provided. Outlet lengths of $e-b=20$ and higher capture the bubble lengths to a precision of at least 5 significant figures.

$N=5$ provides a good accuracy to run the base flow computations. To examine the effect of downstream channel length on the solutions, a convergence study was conducted on the lengths of the first recirculation bubble on the bottom wall of the downstream channel and the secondary recirculation bubble on the top wall. The results shown in Table 2 demonstrate that an outlet length of 10 results in an error in the determination of the primary recirculation bubble length of approximately $0.007 \%$, and approximately $4.5 \%$ for the secondary bubble. The relatively large error in the size of the secondary bubble is caused by its proximity to the outlet. Outlet lengths of 20 to 100 are required to achieve at least 5 significant figures of accuracy. Hence, an outlet length of 30 is considered adequately long to be used throughout this study. Barton (1997) and Cruchaga (1998) studied the entrance effect for backward-facing step flow with expansion ratio of 2 and found that the inlet length of $10 h$ and $2 h$ (where $h$ is the step height), respectively, return slightly different numerical solutions compared to that with zero inlet length. In this study, the inlet length is 15 which is adequately long for the velocity flow to be fully developed before reaching the bend.

\subsection{Test of eigenvalue computations}

The precision of eigenvalue $\mu$ and eigenmode $\hat{\boldsymbol{u}}$ produced by subspace iteration were quantified by the residual

$$
r=\|\mathscr{A} \hat{\boldsymbol{u}}-\mu \hat{\boldsymbol{u}}\|,
$$

where $\|\cdot\|$ was the standard vector norm and where eigenmodes were normalised $(\|\hat{\boldsymbol{u}}\|=1)$. The linear stability analysis technique relied on an iterative process to obtain the leading eigenvalues and eigemodes of the system. The process ceased when $r<10^{-7}$ was achieved. Nevertheless, the eigenmodes are also resolution-dependent. Table 3 reports the accuracy of the eigenvalue computations as a function of element polynomial degree $N$. The leading eigenvalue for $R e=500$, $\beta=1$ and $k=6.4$ is real and linearly stable. It is found that the eigenvalue converged to an error of merely $0.0003180 \%$ at $N=5$, which is employed hereafter. 


$\begin{array}{ccc}N & \left|\mu_{\max }\right| & \text { Relative error } \\ 4 & 0.9941048 & 0.0143794 \% \\ 5 & 0.9942509 & 0.0003180 \% \\ 6 & 0.9942458 & 0.0001927 \% \\ 7 & 0.9942469 & 0.0000837 \% \\ 8 & 0.9942477 & -\end{array}$

Table 3: Dependence of leading eigenvalues on polynomial order. Parameter $N$ indicates the independent polynomial order of the base flow. Leading eigenvalues computed on the mesh at $R e=500, \beta=1$ and spanwise wavenumber $k=6.4$ are provided. The relative error is to the highest polynomial order case $(N=8)$. Given eigenvalues are real.

\subsection{Code validation}

Finally, the present model is validated against published two-dimensional simulations providing the position and length of the recirculation bubble. In a viscous flow (featuring boundary-layers adjacent to no-slip surfaces), the point of reattachment can be precisely measured by finding the location where the wall shear stress $\tau_{\text {wall }}=-\mu \partial u / \partial y$ is zero.

Figure 4 shows a comparison between the recirculation length of the first bubble $\left(L_{\mathrm{R}_{1}}\right)$ in a flow with $\beta=1$ as a function of Reynolds number between the present and previously reported results digitised from figures in Zhang \& Pothérat (2013) and Chung et al. (2003). The coefficient of determination, $R^{2}$, between the present data and those of these previous studies differ by just $2.2 \%$ and $0.2 \%$, respectively. The comparison displays a strong agreement between the studies, with each curve increasing rapidly and linearly at Reynolds numbers $R e \lesssim 200$, before transitioning to a more gradual linear regime of further bubble elongation beyond $R e \approx 300$. This regime terminates with the onset of unsteady flow. The sudden drop in the data from Zhang \& Pothérat (2013) at $R e \approx 600$ coincides with the onset of unsteady flow in that study. The present computations return a steady-state (in agreement with Chung et al., 2003) up to $R e \approx 700$.

\section{Two-dimensional base flows}

\subsection{Flow regimes}

In this section, we focus on the behaviour of the two-dimensional base flow, especially around the sharp bend and along the downstream channel. For the range of opening ratios $\beta$ studied, four regimes are identified (figure 5). The first regime exhibits only a single recirculation bubble immediately behind the sharp bend. The second regime sees the emergence of a bubble at the opposite wall slightly downstream of the first bubble. The third regime reveals the appearance of a small counter-rotating recirculation bubble between the primary recirculation bubble and the bottom wall. Finally, the fourth regime marks the development of an unsteady two-dimensional flow. The Reynolds numbers at the onset of each regime are denoted by $R e_{\mathrm{R}_{1}}, R e_{\mathrm{R}_{2}}, R e_{\mathrm{in}}$ and $R e_{\mathrm{c}}$, respectively as shown in figure 6. The results of the current study agree with those of Zhang \& Pothérat (2013) but small discrepancies are found for $R e_{\text {in }}$ at $\beta \geq 1$, which are attributed to the different nodes density in the meshes at high $R e$ in both studies. The present study also extends the lower end of the range of $\beta$ from $\beta=0.1$ (Zhang \& Pothérat, 2013) to $\beta=0.0125$. The constriction at the bend at smaller $\beta$ leads to high velocities and shear in that region.

For clarity, we shall highlight the main features of the regimes shown in figure 6. but a more detailed description can be found at Zhang \& Pothérat (2013). The onset of all regimes is delayed consistently to larger $R e$ at larger $\beta$ over $\beta \lesssim 1$, and it remains almost constant when $\beta \gtrsim 1$. It is 


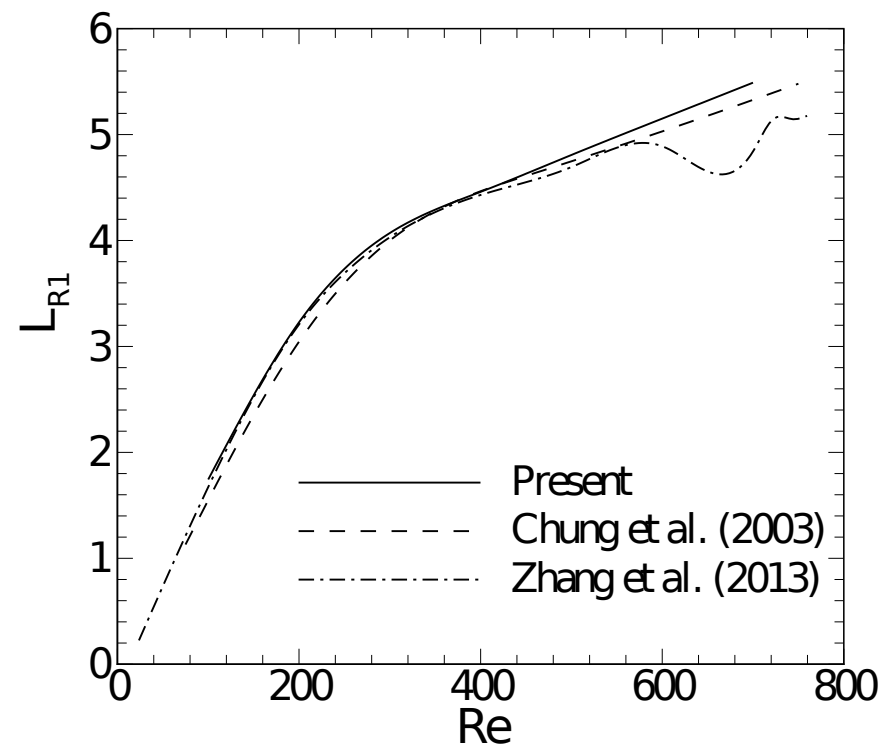

Figure 4: Length of the first recirculation bubble $\left(L_{\mathrm{R}_{1}}\right)$ against Reynolds number $(R e)$ for $\beta=1$, comparing the present results to those of Zhang \& Pothérat (2013) and Chung et al. (2003).

(a) $R e=10$

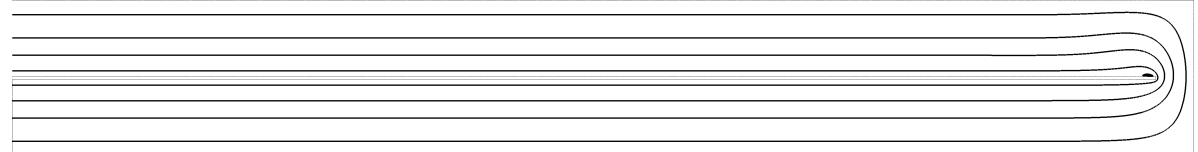

(b) $R e=200$

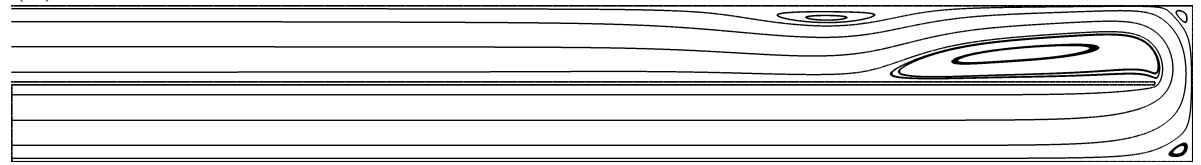

(c) $R e=600$

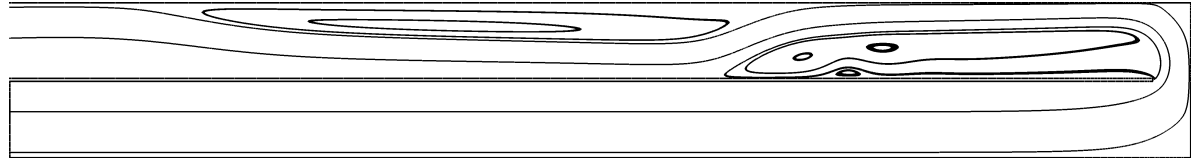

(d) $R e=800$

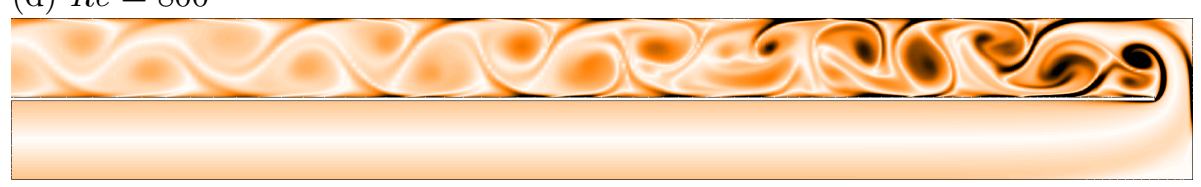

Figure 5: Streamlines of steady two-dimensional base flow at (a) $R e=10$, (b) $R e=200$, (c) $R e=600$ and (d) flooded contours of vorticity magnitude at $R e=800$ for $\beta=0.5$. In (d), white represents zero vorticity (no rotation), and darker shading denotes arbitrarily higher vorticity magnitude levels. 


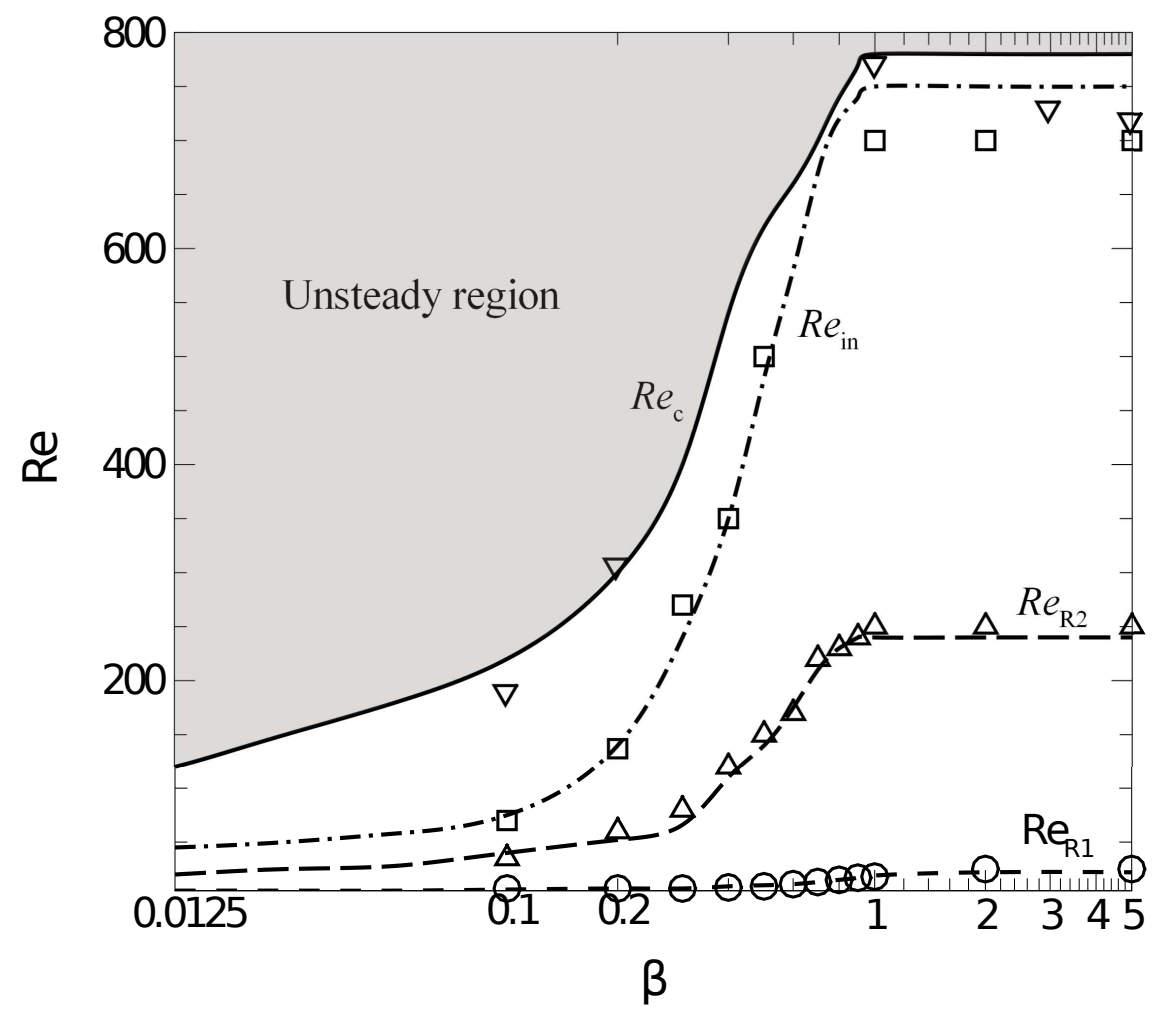

Figure 6: Reynolds number where primary recirculation bubble $R e_{\mathrm{R}_{1}}$, secondary recirculation bubble $R e_{\mathrm{R}_{2}}$ and inside recirculation bubble $R e_{\text {in }}$ start to appear in the two-dimensional flow. $R e_{\mathrm{c}}$ is the transition from steady to unsteady. The lines are from current study and the symbols are results from Zhang \& Pothérat (2013). $\bigcirc, \triangle, \square$ and $\nabla$ represent $R e_{\mathrm{R}_{1}}, R e_{\mathrm{R}_{2}}, R e_{\mathrm{in}}$ and $R e_{\mathrm{c}}$, respectively. 
worth noting that the behaviour of the flow is different between $\beta<0.2,0.2<\beta<1$ and $\beta \gtrsim 1$. For $\beta \lesssim 0.2$; a flow resembling jet flow is created through the narrow bend orifice, causing the flow to accelerate transversely and hit the top wall before deflecting towards the streamwise direction. This causes the onset of each regime to occur at low Re. By contrast, at $0.2 \lesssim \beta \lesssim 1$, the width of the opening of the bend is sufficient for the flow to turn smoothly to the streamwise direction around the bend; hence, $\beta$ influences the onset of each regime. However, at $\beta \gtrsim 1$, the onsets do not vary significantly because a recirculation bubble develops at the outer wall of the bend, which confines the turning flow, such that the true breadth of the bend opening is no longer apparent. Thus the flow behaves similarly to that of $\beta=1$ and the onset $R e$ for the flow regimes remain almost constant for $\beta \gtrsim 1$.

At very low $R e$, the flow in the downstream and upstream channel is almost symmetrical with respect to $y=0$. However, as $R e$ increases, the flow streamlines at the bottom wall move upward until an inflexion point becomes visible behind the edge of the sharp bend. Consequently, the flow separation occurs and a recirculation bubble is formed. In the range of $\beta$ studied, the primary recirculation bubble appears at very small $R e$ because of a strong adverse pressure gradient behind the bend, as can be seen in figure 5(a). Theoretically a sharp edge always causes separation to a flow, even at very low $R e \rightarrow 0$ (Taneda, 1979, Moffatt, 1985). The finite $R e_{\mathrm{R}_{1}}$ captured in this study is a numerical artefact of finite spatial resolution at the sharp bend corner. The finite discretisation obscures the bubble at very low $R e$.

As $R e$ increases, the secondary recirculation bubble appears when the flow streamlines in the bulk flow above the primary recirculation bubble move away from the top wall. This results in a strong adverse pressure gradient at the wall, which causes another separation to occur at $R e_{R_{2}}$ (figure 5(b)). Under the same circumstances, an inner counter-rotating recirculation bubble as seen in figure 5(c), is formed between the primary recirculation bubble and the bottom wall when the backflow in the primary recirculation bubble moves away from the wall.

Zhang \& Pothérat (2013) found an additional regime between $R e_{\text {in }}$ and $R e_{\mathrm{c}}$, where they reported a small scale vortices structure far downstream of the channel; this state is not observed in the current study. $R e_{\mathrm{c}}$ in figure 6 indicates an unsteady flow where large eddies or vortices are shed downstream from the sharp corner of the bend as depicted in figure $5(\mathrm{~d}) . R e_{\mathrm{c}}$ is found to be in agreement with those of Zhang \& Pothérat (2013) study, and it is important to note that both studies started simulations from rest to obtain this $R e_{\mathrm{c}}$. In a further analysis, hysteretic behaviour has been observed when the simulation is started from different initial conditions. Considering the unsteady flow as a departure from the base steady flow at the same Reynolds number, its amplitude $|A|$ is measured as the $\mathscr{L}^{2}$ norm (the integral of the magnitude of velocity over the computational domain) of the difference between velocity fields in these states. Figure 7 shows the variations of $|A|$ and therefore regimes of unsteady flow where $|A|>0$ when Reynolds number is incrementally varied for $\beta=1$. Two distinct onsets of two-dimensional unsteadiness are found by initiating the simulation from three different initial conditions which are (i) flow at rest, (ii) a snapshot of unsteady flow computed at a slightly lower Reynolds number, and (iii) the steady flow solution obtained at a slightly lower Reynolds number. It is evident from the figure that the simulations starting from the flow at rest and from an unsteady flow yield the same value of $R e_{\mathrm{c}}=742$, whereas the simulations starting from a steady flow become unsteady at a higher $R e$, $R e_{\mathrm{c}} \approx 1150$.

The location where shedding initiates at the onset depends on initial conditions too. When a simulation is initiated from a flow at rest, the vortex shedding can be seen to emerge from the sharp corner of the bend as illustrated in figure 8(a). It is likely that a large-amplitude perturbations caused by the impulsive initiation of the flow are sufficient to provoke a shedding from the bend that bypasses the downstream destabilisation, and beyond $R e_{\mathrm{c}} \approx 742$ (at least in these simulations) is self-sustaining. A similar observation was made when reducing Re from an unsteady flow: a 


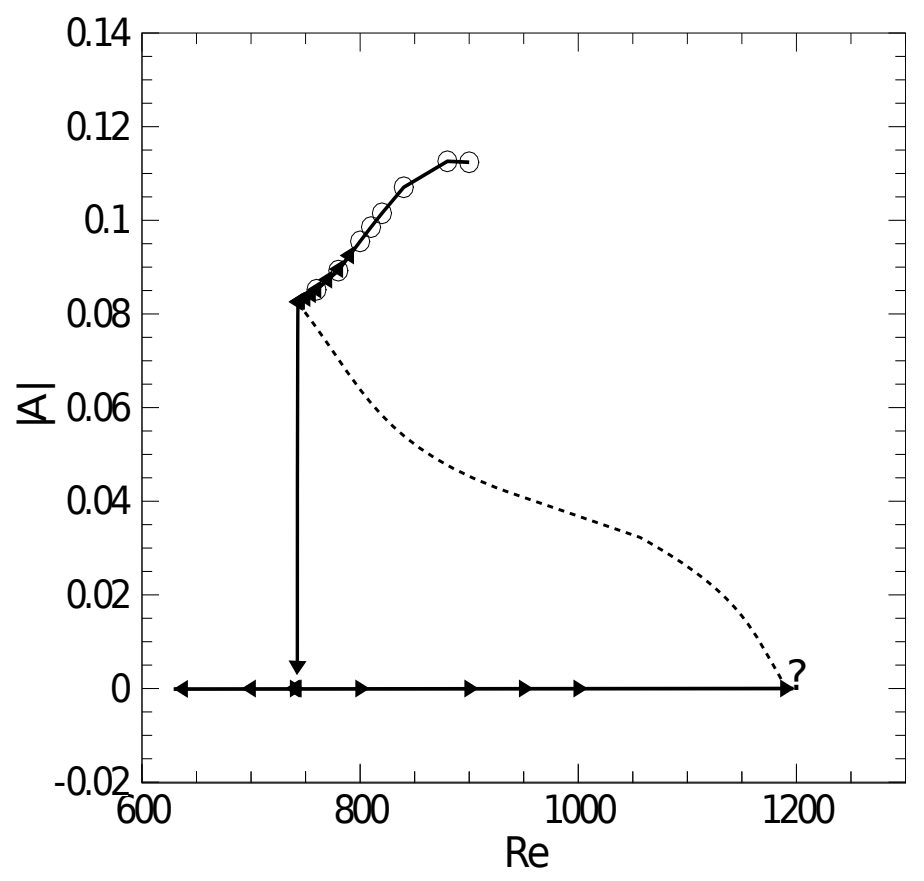

Figure 7: Hysteretic behavior described by the fluctuation of the integral of velocity magnitude throughout the domain as a function of $R e$ at $\beta=1$, reducing $R e$ from an unsteady flow (४) and increasing $R e$ from a steady flow $(\checkmark)$ give different $R e_{\mathrm{c}} \cdot|A|=0$ indicates steady flow solution. 


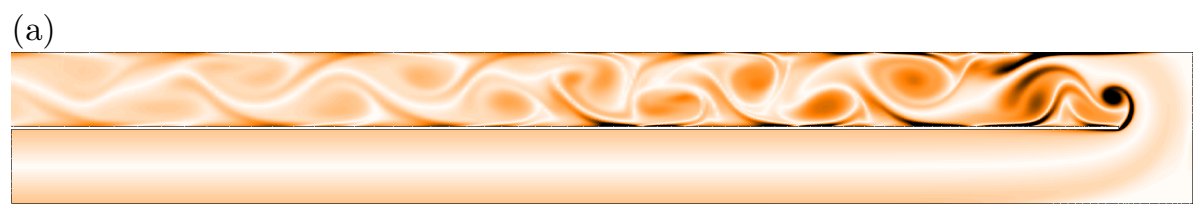

(b)

(c)

(d)

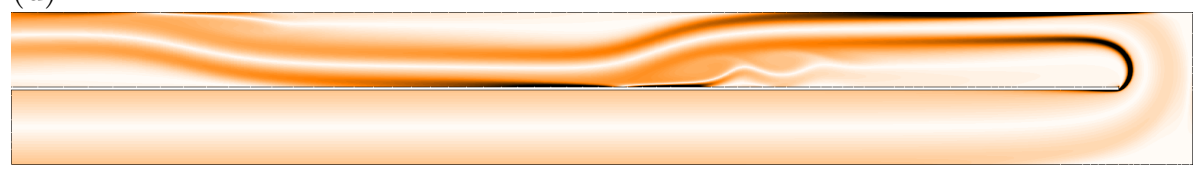

Figure 8: Flooded contours of vorticity magnitude demonstrating unsteady saturated flows for (a) $R e=800$ at $\beta=1$ (which was initiated from rest), and (b)-(d) $R e=1152$ at $\beta=1$ (which was initiated from a saturated steady-state flow solution at a lower Reynolds number). Contour levels are as per figure 5(d). (b), (c) and (d) show domain segments $-45 \leq x \leq-29,-30 \leq x \leq-14$ and $-15 \leq x \leq 1$, respectively.

large sudden decrease could revert the unsteady flow to steady state at a Reynolds number at which the unsteady state could be preserved via a gradual decrement in Reynolds number.

When $R e$ is increased gradually along the steady-flow branch, the flow remains steady up to $R e \simeq 1150$. Figure 8(b)-(d) depicts the saturated flow at $R e=1152$. This final state exhibits a wavy disturbance extending 25 diameters downstream of the bend. In this case, unsteadiness first manifested in the shear layers behind the secondary recirculation bubble in the form of small eddies, but over time the flow in this region more proximate to the bend re-stabilised and the unsteady region retreated to its ultimate position further downstream. This has some resemblance to the regime described by Zhang \& Pothérat (2013) before the flow becomes unsteady in their study. It is likely that they found this regime at lower $R e$ due to the high sensitivity to mesh resolution of this feature. Since the flow is already unstable far downstream of the bend, noise tended to be amplified and created small vortical structures. In testing this hypothesis, we found that by increasing resolution of the mesh in our study, the onset of unsteadiness could be delayed significantly by increasing $R e$ gradually. It is also plausible that the length of the outlet channel may influence the upper limit of the steady-state branch, though this was not tested. Finally, it is noted that when comparing the flow pattern and the region of the flow producing the unsteady flow features between figure $8(\mathrm{a})$ and figure $8(\mathrm{~b})-(\mathrm{d})$ that the two depicted unsteady branches are different. It will be shown in $\S 5$ that the two-dimensional flows are unstable to three-dimensional perturbations at Reynolds numbers below those of this hysteretic zone, so it will not be characterised further here. 


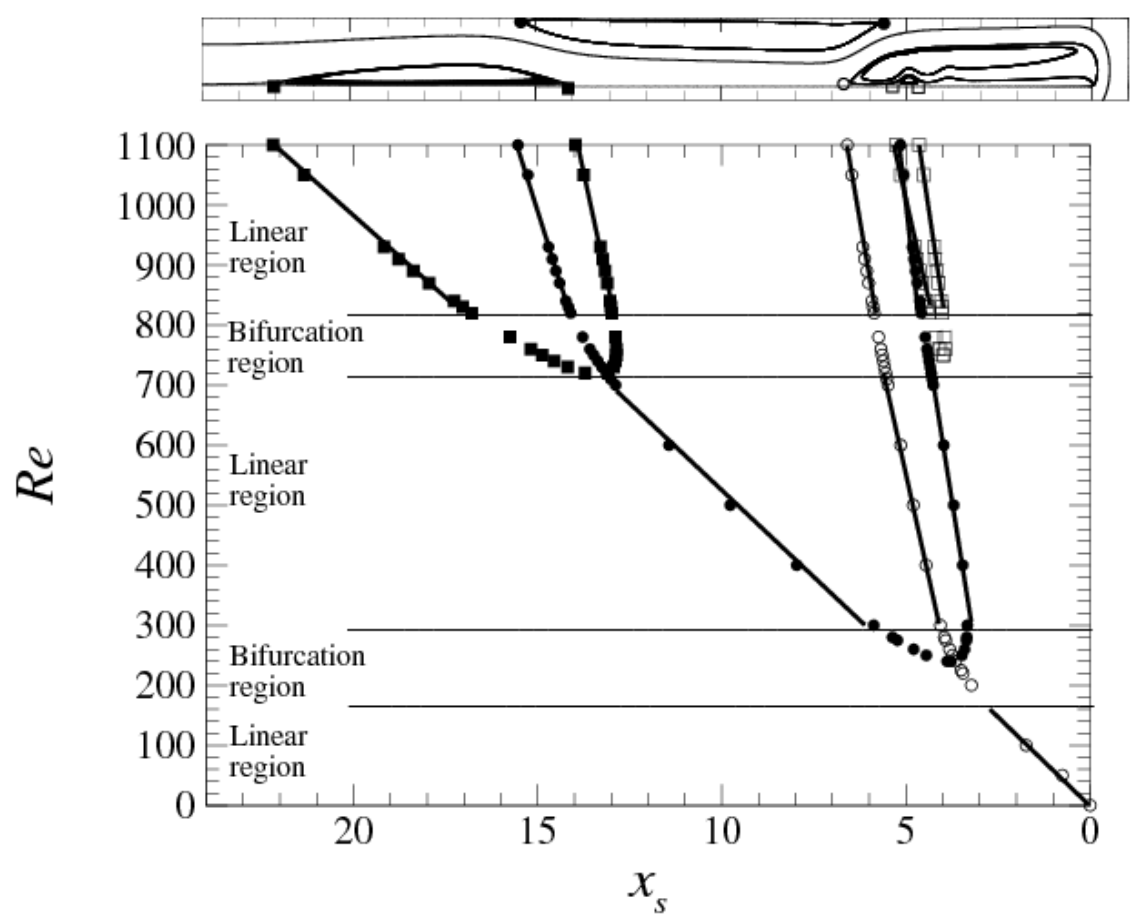

Figure 9: A plot showing the Reynolds-number-dependence of the locations of separation points (measured by their distances from the bend - left of the origin), $x_{s}$, for the base flows as functions of Reynolds number. Open circles represent the stagnation points for the primary recirculation bubbles. Solid circles denote the stagnation points for the secondary recirculation bubbles which forms at $R e \approx 240$ and $x_{s} \approx 3.77$. Solid and open squares represent the stagnation points for third bubble and inner recirculation bubble, respectively, which are formed at higher $R e$. The top frame shows the streamlines of the base flow and the separation points of primary, secondary, inner and third recirculation bubbles at $R e=1100$ in the outlet channel.

\subsection{Bubble separation points (steady flows)}

Figure 9 shows the Reynolds number dependence of the separation and reattachment points (expressed by their distance downstream of the bend, $x_{s}$ ) on both the bottom and top walls of the channel for $\beta=1$. The empty circle symbol indicates the limit of the primary recirculation bubble behind the sharp corner. At $R e=240$, a secondary recirculation bubble appears on the top wall at $x_{s}=3.77$. At $R e \approx 700$, two additional recirculation bubbles appear at the bottom wall; one is a small bubble in the primary recirculation bubble and the other is near the reattachment point of the secondary recirculation bubble.

From figure 9, we notice that there are regions where the location of the separation and reattachment points are almost linear functions of $R e$. It can clearly be seen in figure 9 that when the bifurcation region around $R e=300$ and 750 is being excluded, the locations of both points for all bubbles behave almost linearly with $R e$. When a new recirculation bubble appears, the growth of the recirculation bubble just upstream of it is affected and so is the variation with Reynolds of 


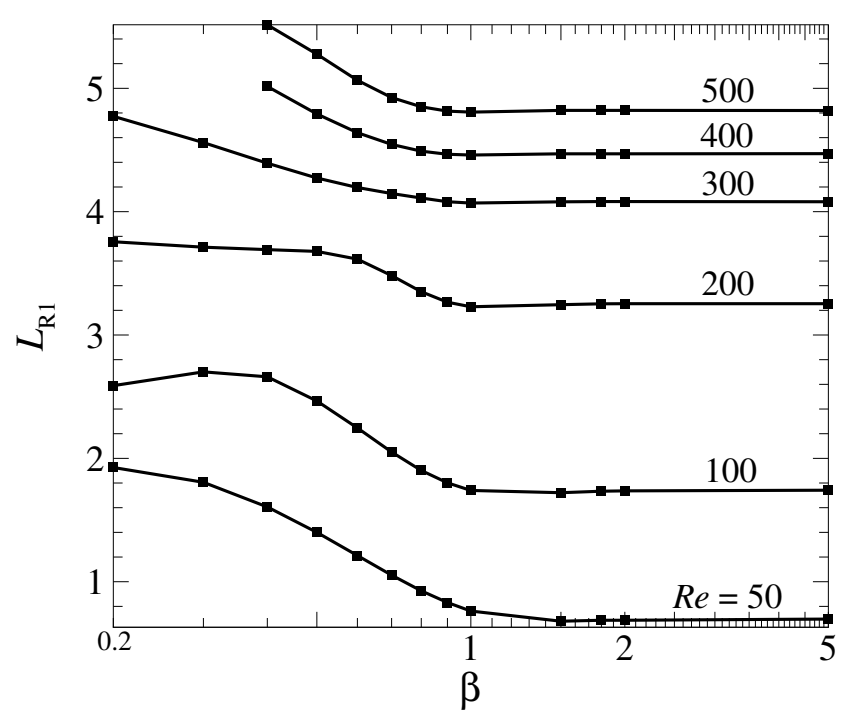

Figure 10: A plot of the length of the primary recirculation bubble as a function of $\beta$ for Reynolds numbers as labelled.

its reattachment point. The same behaviour was also found in the backward-facing step flow by Erturk (2008).

The effect of $\beta$ on the size of the primary recirculation bubble is illustrated in figure 10. At small values of $\beta$, the main bulk flow is accelerated by the small jet opening, making the primary recirculation bubble elongated in the streamwise direction. This causes the bubble to be bigger than that at larger $\beta$. $L_{\mathrm{R} 1}$ decreases as $\beta$ increases, but as $\beta>1$ and $R e \gtrsim 200$, the size of the bubble increases due to the effect of the recirculation bubble at the far end of the bend wall.

Since for $\beta>1$, part of the flow in the bend is trapped in a closed eddy outside of the throughflow taking the bend, Zhang \& Pothérat (2013) defined an effective opening ratio $\beta_{\text {eff }}$ based on the horizontal thickness of the flow effectively turning from inlet to outlet at $y=0-$ that is, the distance from the inner vertical wall at the bend to the dividing streamline separating the turning flow from the closed recirculation. The same definition is used in the present study. For $\beta \leq 1$,

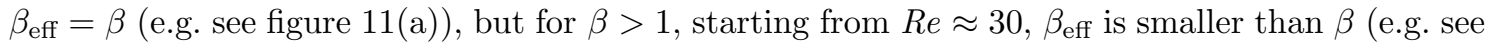
figure $11(\mathrm{~b}))$. At $R e>200, \beta_{\text {eff }}$ saturated close to 0.7 . This degradation of $\beta_{\text {eff }}$ to values below $\beta$, and its saturation behaviour at large $R e$, can be observed in figure 11(c). For $\beta=2$ in figure 11(b), the effective $\beta$ is found to be $\beta_{\text {eff }} \approx 0.8$ which is slightly higher than what was found by Zhang \& Pothérat (2013).

\section{$5 \quad$ Linear stability}

\subsection{Growth rates and marginal stability}

This subsection analyses the dependence of the perturbation growth on Reynolds number $R e$, spanwise wavenumber $k$ and opening bend ratio $\beta$. Figure 12 shows the predicted growth rates as a function of the Reynolds number and spanwise wavenumber $k$ for $\beta=0.2,0.5,1$ and 2 . The primary linear spanwise instability is obtained via polynomial interpolation to determine the 


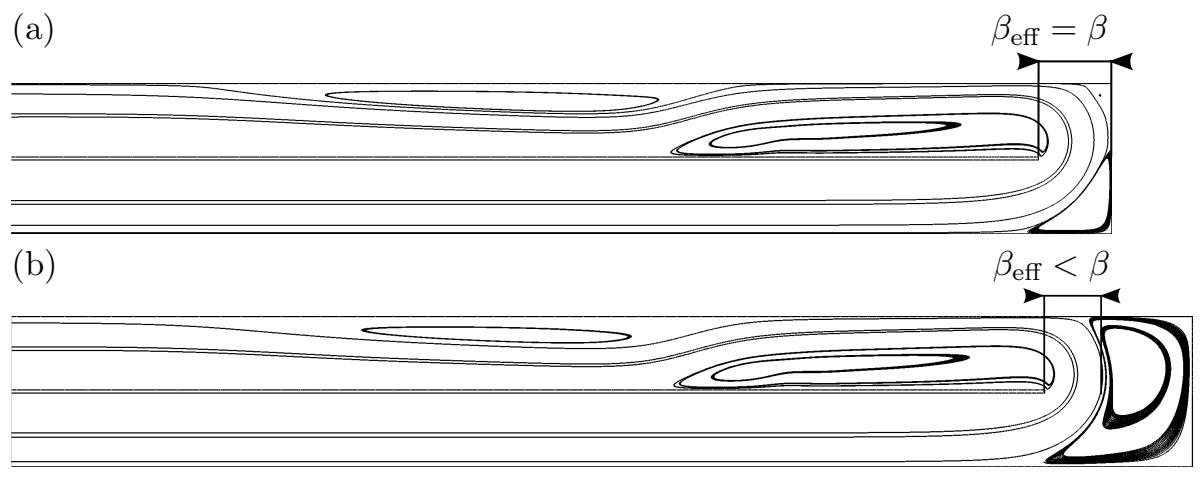

(c)

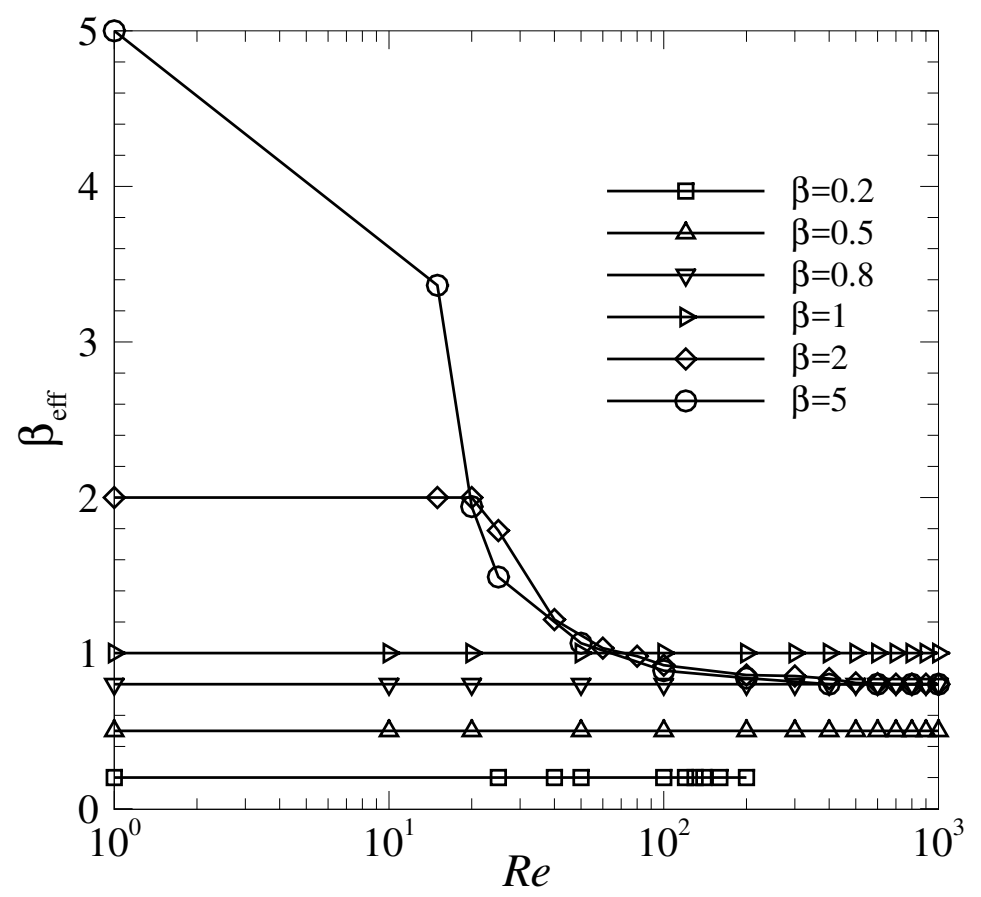

Figure 11: Streamlines of steady two-dimensional flow for $R e=600$, (a) $\beta=1$ and (b) $\beta=2$. A big recirculation bubble appears at the far end of the wall when $\beta>1$ causing the effective bend opening ratio to be lesser than the actual opening. $\beta_{\text {eff }}(R e)$ is plotted in (c) for several value of $\beta$. 


$\begin{array}{ccc}\beta & R e_{\mathrm{c}} & k_{c} \\ 0.2 & 125 & 3 \\ 0.5 & 278 & 2.05 \\ 1 & 397 & 1.98 \\ 2 & 387 & 1.93\end{array}$

Table 4: Critical Reynolds number and corresponding spanwise wavenumber at the onset of instability for $\beta=0.2,0.5,1$ and 2 .

lowest Reynolds number that first produces $\sigma=0$, and the wavenumber at which this occurs. Both are reported in table 4 . For $\beta>0.2$, at very low wavenumber $k \lesssim 0.3$, a local maximum is observed, but the corresponding mode is always stable. Between this local maximum and the primary maximum, a small range of $k$ produces leading non-real eigenvalues. Larger wavenumbers that those shown in figure 12 were also studied and found decay increasingly fast at large $k$. This trend of the growth rate as a function of $R e$ and $k$ shows a good resemblance with those of backward-facing step flow (Barkley et al., 2002). However, for all $\beta$, the critical Re for the flow to become three-dimensional are found to be much lower compared to the flow in backward-facing step (Barkley et al., 2002, Armaly et al. , 1983) and partially blocked channel (Griffith et al., 2007). This finding is not surprising, as the two-dimensional flow around sharp bend becomes unsteady at much lower $R e$ compared to those geometries.

Figure 12 shows that $\beta=0.2$ always has an oscillatory leading mode for the range of Re investigated. Meanwhile, $\beta=0.5$ has a synchronous leading mode at $R e \leq 400$, before an oscillatory mode becomes dominant at higher Re. On the other hand, $\beta>1$ always has a synchronous leading mode. The transition from oscillatory to synchronous behaviour as $\beta$ increases is similar to what was observed by Lanzerstorfer \& Kuhlmann (2012) in backward facing step flow, where they found that the the flow with very large step height is destabilised by an oscillatory mode, and this changes from oscillatory to synchronous if the step height is further decreased. To clarify the shift of the leading mode from synchronous to oscillatory, several of the leading eigenvalues have been computed for $\beta=0.5$ at each wavenumber and three different Reynolds numbers.

The results are shown in figure 13. The curves closely resemble those for the flow over a backward-facing step (Barkley et al., 2002), which consists of two branches of real eigenvalues at low wavenumber coalescing into a single branch of non-real eigenvalue as $k$ increases. In Barkley et al. (2002)'s case, however, the primary leading eigenvalues appear at higher wavenumbers, and (as with the present study for $\beta>0.5$ ) the real branch at high wavenumber is the first to become unstable. All branches shift to higher $\sigma$ as Re increases. However, as Re increases further, it can be seen that the leading oscillatory mode is more stable than the real one (figure 13(b)) before it becomes dominant at $R e \approx 600$ as shown in figure 13(c). A similar observation was made by Natarajan \& Acrivos (1993) in flow past spheres and disks, by Tomboulides \& Orszag (2000) in the weak turbulent flow past a sphere, and by Johnson \& Patel (1999) in a numerical and experimental study on flow past a sphere up to $R e_{d}=300$.

\subsection{Structure of the eigenvalue spectra}

Figure $14(\mathrm{a}, \mathrm{d}, \mathrm{g})$ show the eigenvalue spectra for three different cases. Each has a different type of leading eigenmode, and the dashed line indicates the onset of instability $(\sigma=0)$. The real part of the eigenmodes are visualised via plots of spanwise vorticity on the plane $z=0$ in figure 14(b, $\mathrm{c}, \mathrm{e}, \mathrm{f}, \mathrm{h}, \mathrm{i}$ ). Figure 14 (a) depicts the eigenvalue spectrum for $\beta=0.2, R e=120$ and $k=2.8$, which is near to the onset of instability. Two complex-conjugate pairs of non-real eigenvalues are the fastest-growing eigenvalues in the spectrum. The leading pair (e.g. figure 14(b)) exhibits a 
(a)

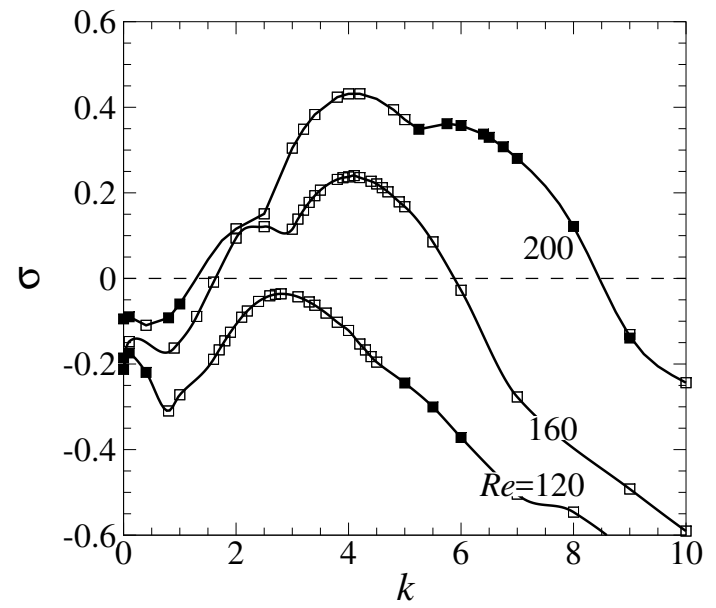

(c)

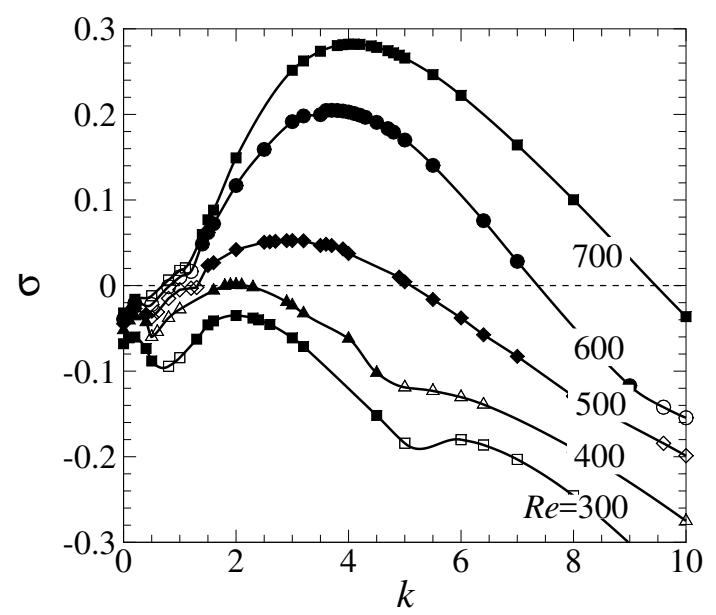

(b)

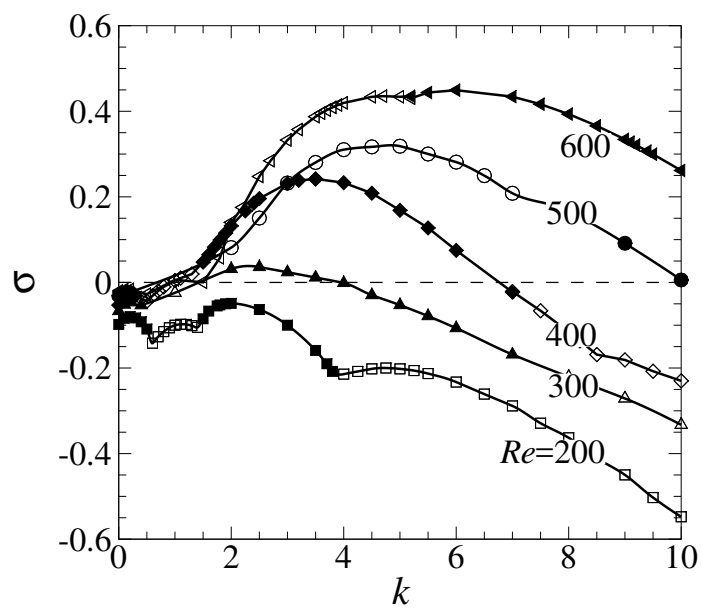

(d)

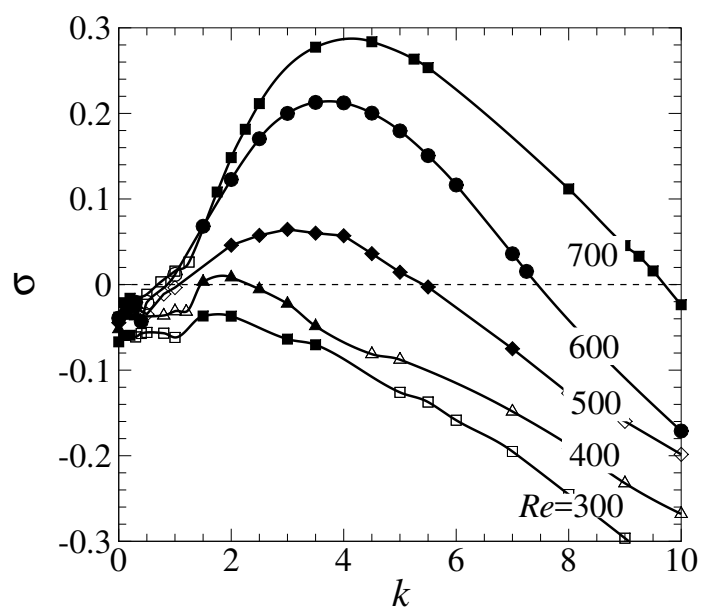

Figure 12: Growth rates of leading eigenmodes as a function of spanwise wavenumber $k$ for (a) $\beta=0.2$ and $R e \leq 200$, (b) $\beta=0.5$ and $R e \leq 600$, (c) $\beta=1$ and $R e \leq 700$, and (d) $\beta=2$ and $R e \leq 700$. Solid symbols represent real leading eigenvalues, while hollow symbols represent complex-conjugate pairs of non-real leading eigenvalues. Solid lines connect all dominant leading eigenvalues from several branches of the same Reynolds number. 
(a)

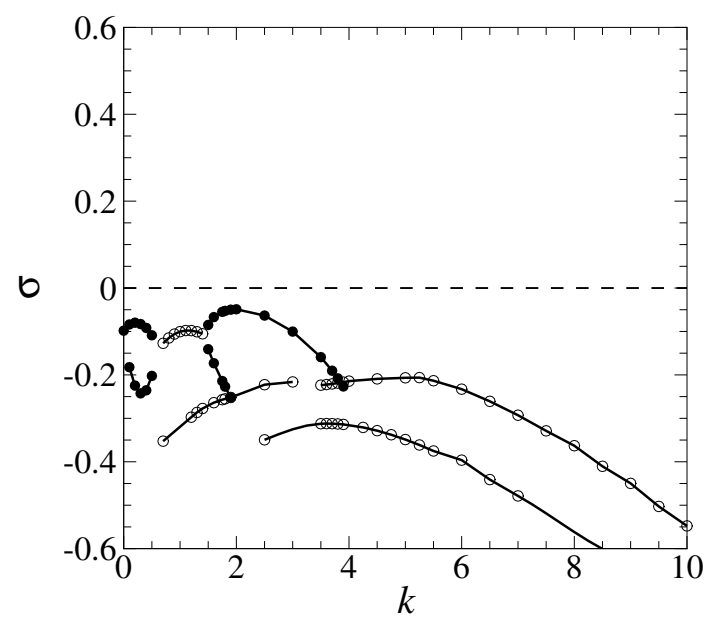

(b)

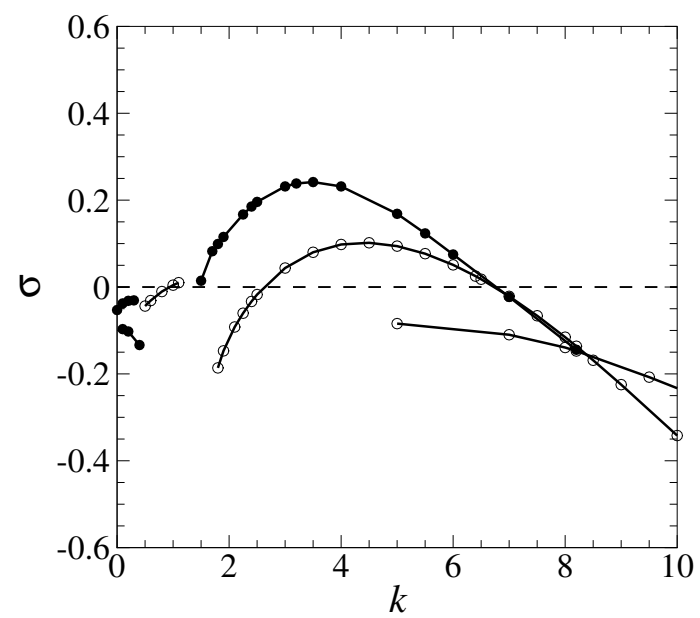

(c)

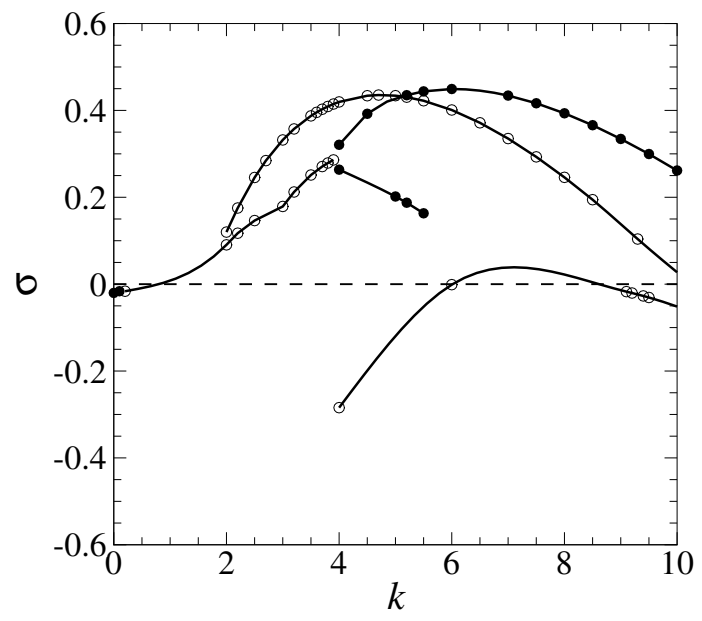

Figure 13: Growth rates of leading eigenmodes plotted against spanwise wavenumber at $\beta=0.5$ for (a) $R e=200$, (b) $R e=400$ and (c) $R e=600$. Solid symbols represent real leading eigenvalues, meanwhile hollow symbols represent complex-conjugate pairs of leading non-real eigenvalues. 
(a)

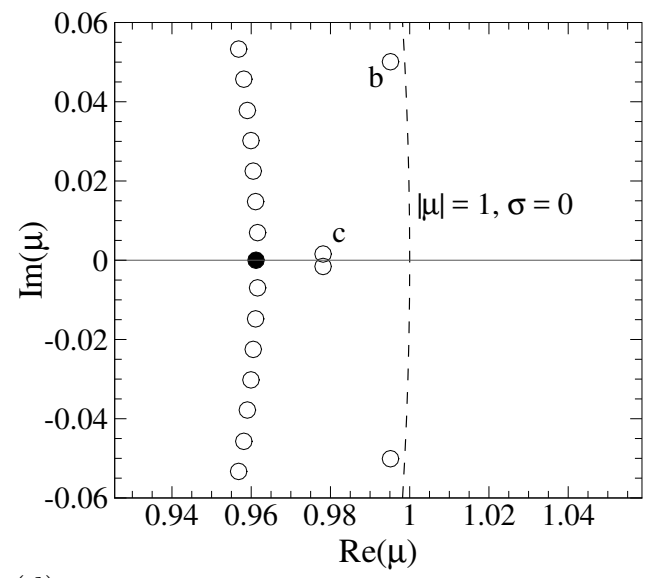

(b)

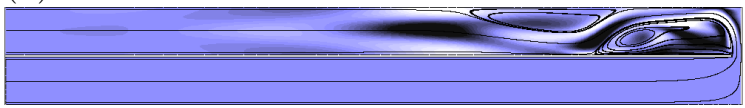

(c)

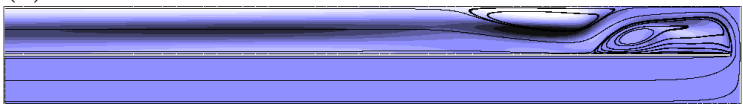

(d)

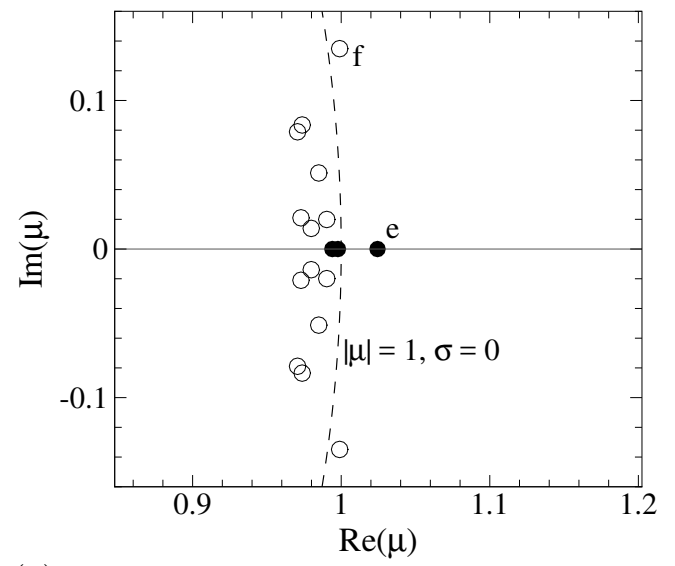

(e)

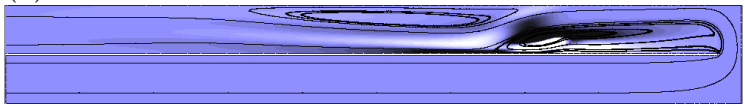

(f)

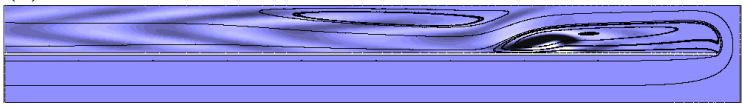

(g)

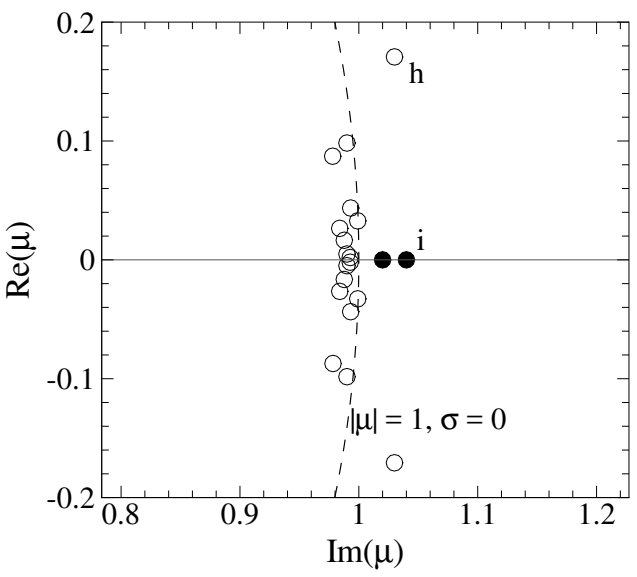

(h)

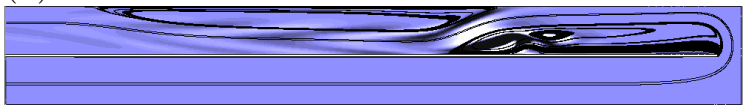

(i)

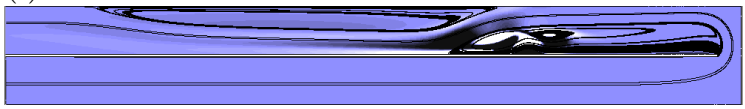

Figure 14: Eigenvalue spectra for (a) $\beta=0.2, R e=120, k=2.8$, (d) $\beta=0.5, R e=400, k=3.5$, and $(\mathrm{g}) \beta=0.5, R e=600$ and $k=4.5$. (b, c, e, f, h, i) visualise the real part of the complex eigenvector fields via spanwise vorticity on the plane at $z=0$. (b), (e) and (h) show the respective leading eigenvalues, while (c), (f) and (i) show the corresponding field for the second most dominant eigenmode. In these vorticity plots, zero vorticity is shown by the mid-level shading, while darker and lighter shading respectively show negative and positive shading. 
(a)

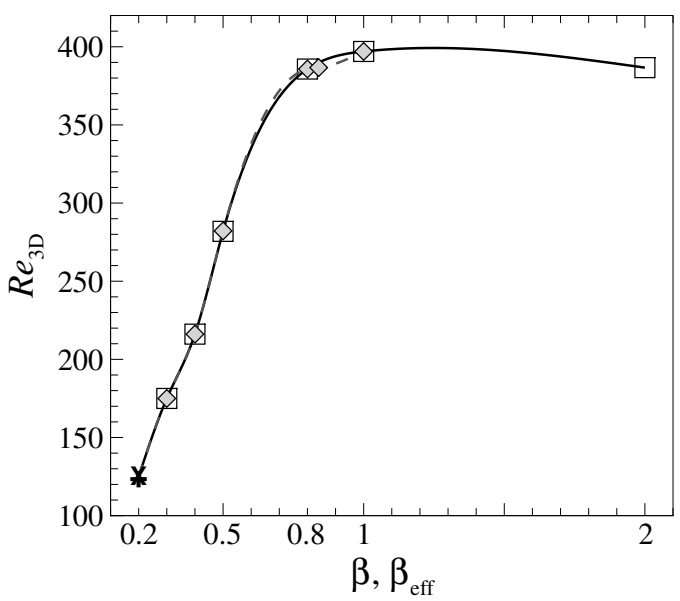

(b)

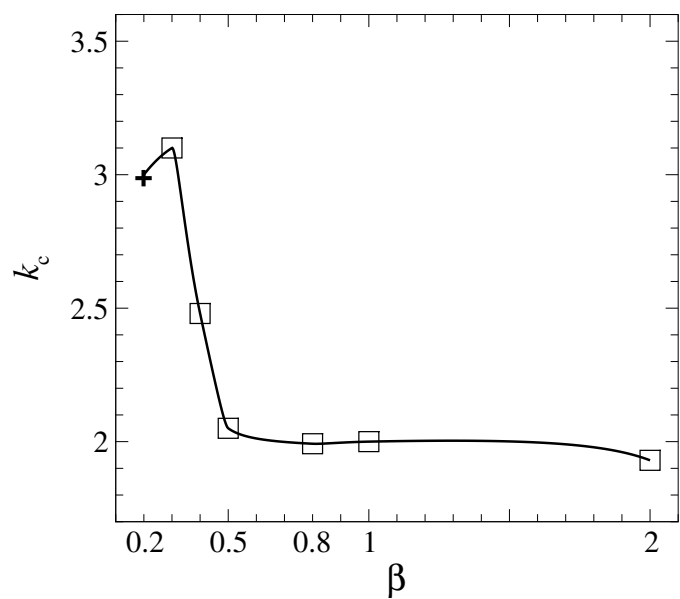

Figure 15: Critical (a) Reynolds number and (b) wavenumber as a function of opening ratio. In (a) the critical Reynolds number is also plotted against effective opening ratio $\beta_{\text {eff }}$. For $\beta$ data, synchronous modes and oscillatory modes are represented respectively by " $\square$ " and "+". For $\beta_{\text {eff }}$ data, synchronous and oscillatory modes are respectively represented by "ऽ" and " $\times "$.

strong growth rate, with strong perturbation structure in the primary recirculation bubble, while the second pair (e.g. figure 14(c)) has perturbation structure mainly localised in the bulk flow near the secondary recirculation bubble.

In contrast to $\beta=0.2$, larger $\beta$ tend to favour synchronous leading modes. $\beta=0.5$ is particularly interesting because this is the point a transition from synchronous ( $R e=400$ in figure 14 (d)) to oscillatory $(R e=600$ in figure $14(\mathrm{~g}))$ leading mode is seen. The leading eigenvalue for $R e=400$ is synchronous, while the second-largest eigenmode is oscillatory. However, at higher $R e$, the oscillatory mode has higher growth rate compared to the synchronous mode, as can be seen in figure $14(\mathrm{~g})$. The perturbation fields associated with these modes are strongest in the same vicinity, which is in the primary recirculation bubble near to the reattachment point. From the contours represented in figures 14(h) and (i), we can see that the contours in figure 14(b), 14(e) and 14(i) have a similar synchronous eigenmode structure; meanwhile figures 14 (c), 14(f) and 14(h) have a consistent oscillatory eigenmode structure. The oscillatory mode structure is distinguished from the synchronous mode structure by the presence of an array of chevron-shaped vorticity structures following the core flow downstream from the aft end of the primary recirculation bubble.

\subsection{Dependence on $\beta$ and analogy with related flows}

From the known influence of the expansion ratio to the three-dimensional characteristics of backwardfacing step (Barkley et al., 2002, Lanzerstorfer \& Kuhlmann, 2012) and opening ratio in partially blocked channel (Griffith et al. 2007 ) and to the two-dimensional characteristics of 180-degree sharp bend (Zhang \& Pothérat, 2013), we shall expect that the three-dimensional flow in a 180degree sharp bend flow is also dependant on the same physical parameter (here $\beta$ ).

Figure 15illustrates the effect of $\beta$ on the $R e$ and $k$ at the onset of three-dimensional instability. In the range of $\beta$ studied, only the case $\beta=0.2$ becomes three-dimensional through the onset of an oscillatory mode; while all other cases transition through the onset of a non-oscillatory mode. Apparently, $R e_{3 \mathrm{D}}$ increases steadily as the bend opening becomes larger until $\beta \approx 1$. $R e_{3 \mathrm{D}}$ at 


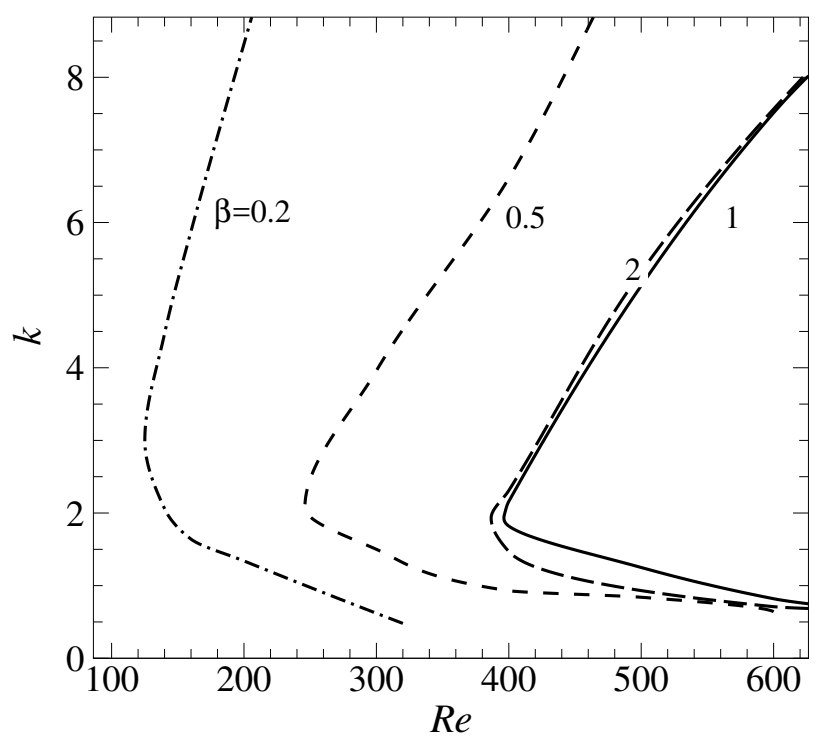

Figure 16: Marginal stability curves for sharp 180-degree bend flow with $\beta=0.2,0.5,1$, and 2 . Regions on the right of the curves represent flow conditions that are linearly unstable to threedimensional perturbations for that particular $\beta$.

$\beta=2$ is lower than $\beta=1$ because of the appearance of the recirculation bubble at the far end of the bend wall that limits the width of the bulk flow in the bend, causing a reduction in effective value of $\beta$. If we look closely, the value of $R e_{3 \mathrm{D}}$ at $\beta=2$ is almost the same as that of $\beta=0.8$, which testifies to the importance of $\beta_{\text {eff }}$ in determining the stability of the flow. In figure 15 (a), $R e_{3 \mathrm{D}}$ is also plotted against $\beta_{\text {eff }}$ to illustrate this behaviour.

The eigenvector fields at the onset of instability for all synchronous modes $(\beta \gtrsim 0.3)$ have perturbation structure located in the recirculation bubble, similar to what was found in the backwardfacing step flow (Barkley et al. , 2002, Alam \& Sandham, 2000), partially blocked channel flow (Griffith et al., 2007) and separation flow (Hammond \& Redekopp, 1998). Barkley et al. (2002) concluded that the size and shape of the bubble directly affected the instability. Conversely, Hammond \& Redekopp (1998) and Alam \& Sandham (2000) confirmed in their studies that the onset of local absolute instability depended on the backflow of the bubble. Interestingly, $\beta=0.8$ and $\beta=2$ have comparable bubble size, peak backflow velocity and location of the peak backflow velocity (with discrepancies of only $4.3 \%, 1.0 \%$ and $0.4 \%$, respectively). This supports the view that for $\beta>1$, the stability of the flow is characterised by $\beta_{\text {eff }}$, in both of these cases $\beta_{\text {eff }}=0.8$.

The dependence of $k_{c}$ on $\beta$ is also illustrated in figure 15 . As $\beta$ increases from 0.3 to 0.5 , the dominant wavelength of the instability increases from $\lambda \approx 2 \pi / 3$ to $\approx \pi$. This is perhaps due to the transition from jet-like flow around the bend to a broader turning flow. $k_{c}$ at $\beta=0.2$ does not follow the trend observed between $\beta=0.3$ and 0.5 , due to the different mechanism. For $\beta \geq 0.5$, the critical wavenumber exhibits little dependence on $\beta$.

Figure 16 shows the marginal stability curves at several $\beta$. The marginal curves are obtained by interpolating $\sigma(k, R e)$ to zero growth rate for each $R e$ from figure 12 . For $\beta \leq 1$, with increasing $\beta$ the neutral stability curve shifts to the right as expected as the flow with wider bend opening ratio is more stable than those with smaller opening ratios. Beyond $\beta \approx 1$, the stability curve recesses slightly towards lower Reynolds numbers, occupying the region between the marginal stability 
(a)

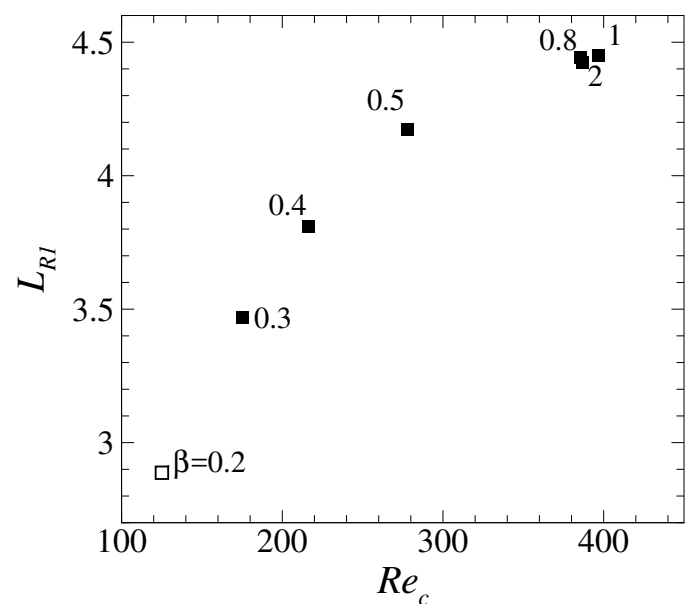

(b)

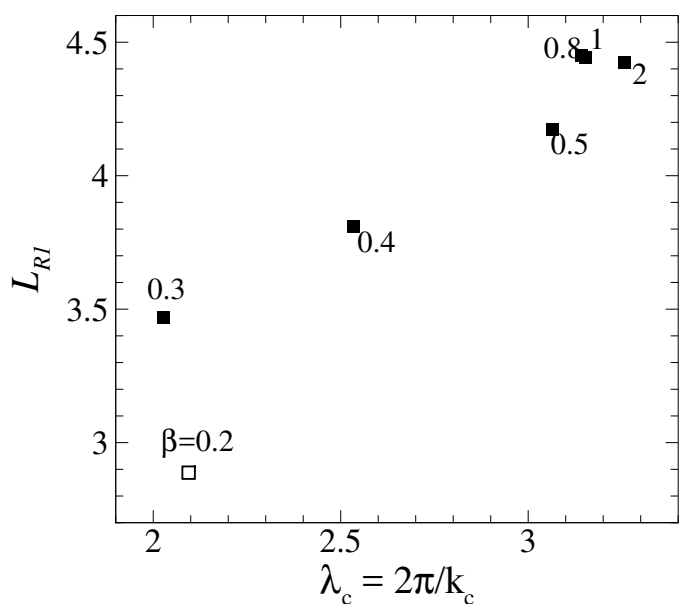

Figure 17: Length of primary recirculation bubble as a function of (a) critical Reynolds number and (b) critical wavelength.

curves for $\beta=0.5$ and 1 . This is explained by the decrease in $\beta_{\text {eff }}$ with $\beta$ for $\beta>1$ (see $\S 4$ ). The stability curves also show a decrease in dominant wavenumber with increasing $\beta$. This is because the instability is scaled with bubble size as discussed by Barkley \& Henderson (1996). The length of the primary recirculation bubble as a function of critical Reynolds number and critical wavelength is depicted in figure 17. It can be seen from figure 17 (a) that the flow becomes unstable at higher $R e$ at bigger $\beta$. For the synchronous modes $(0.3 \lesssim \beta \lesssim 1)$, the critical wavelength increases as the size of the primary recirculation bubble increases.

In order to consider the three-dimensional stability of these flows in the context of the underlying two-dimensional flows, figure 18 summarizes the $(R e, \beta)$ parameter space explored. Within this range, instability to three-dimensional perturbations always occurs in the regime where both primary and secondary recirculation bubbles exist. This agrees with Armaly et al. (1983), who found for the backward-facing step that three-dimensionality appeared in the flow after the secondary recirculation bubble had formed. In our study, $R e_{3 \mathrm{D}}$ increases monotonically with $\beta$ before slightly reducing and becoming independent on $\beta$ as $\beta$ exceeds unity. The critical eigenvalue for $\beta=0.2$ is found to be non-real, while it is real for $\beta=0.5,1$ and 2. Across all considered Re studied at $\beta=0.2$, the dominant eigenvalues are non-real. On the other hand, for $\beta \gtrsim 1$, the dominant eigenvalues are consistently real. A transition from real to non-real (from solid to hollow symbol) can be seen at $\beta=0.5$ in figure 18 which occurs near the regime where the inside recirculation appears in the primary recirculation bubble.

\subsection{Instability mode structure}

In this section, the mechanisms by which the three-dimensional infinitesimal perturbations are amplified are addressed. The obvious mechanism seen in the two-dimensional flow is KelvinHelmholtz instability. Zhang \& Pothérat (2013) found that in regime IV, the shear layers around both bubbles are subject to it, and lead to unsteadiness. However, as this study demonstrates, absolute three-dimensional instabilities are found at $R e \ll R e_{\mathrm{c}}$ involving different mechanisms.

As mentioned earlier, the structure of instability affecting the primary recirculation bubble bears a strong similarity to both the flow over a backward-facing step and in a partially blocked 


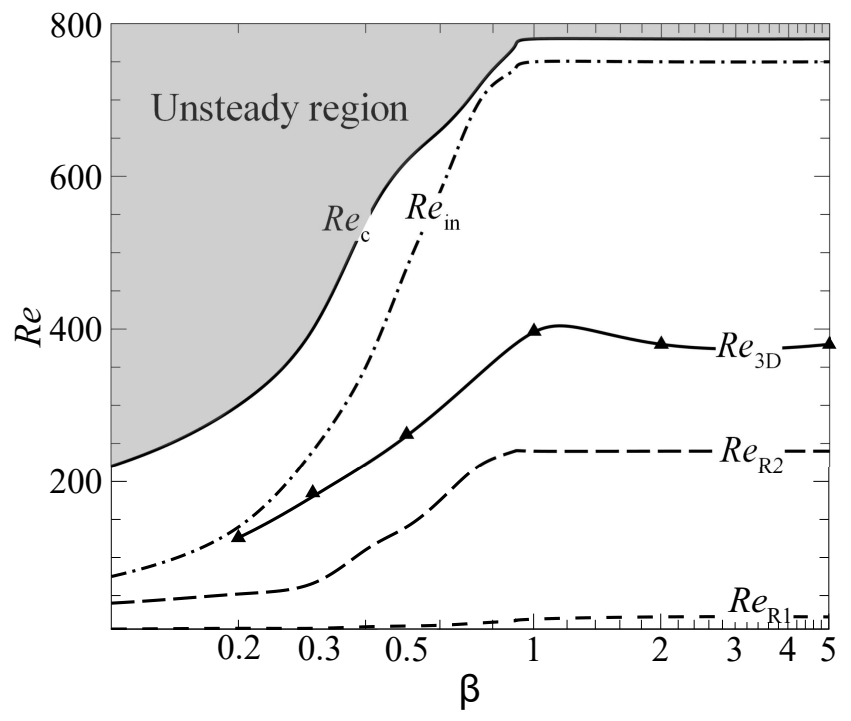

Figure 18: Parameter space for flow regimes and leading peak eigenvalues of three-dimensional stability. The onset of three-dimensional instability is represented by $\triangle$ symbols. Lines are included for guidance, and the shaded region shows the parameter values exhibiting unsteady two-dimensional solutions.

channel. Ghia et al. (1989) suggested that the appearance of the secondary bubble introduced a concave curvature in the streamlines of the bulk flow, thus inducing Taylor-Görtler instability. However, this scenario has been ruled out (Barkley et al., 2002, Griffith et al., 2007) because instability arises neither in the secondary recirculation bubble nor in the main bulk flow between the primary and secondary recirculation bubble zones. The leading instability mode from our analysis is also localised in a different location; though the second-leading lower-wavenumber eigenmode is found to be located in these regions.

Figure 19 visualises the real part of the leading eigenmode at gap ratios spanning $0.2 \leq \beta \leq 2$. Three-dimensionality appears at the reattachment and separating points of the primary recirculation bubble as shown by the spanwise velocity component in figure 19(b)(iii), (c)(iii) and (d)(iii). The perturbation is strongest within the closed streamlines of the primary recirculation bubble (ref. the isosurface plots in figure 19p, with further perturbation structure also present in the secondary bubble and propagating downstream into the core jet flow.

Spanwise perturbation vorticity contour plots shown in figure 19 exhibit perturbation vorticity structures that resemble those arising from an elliptic instability, namely a pair of counter-rotating vortices inside the recirculation bubble. A similar interaction of counter-rotating vortices in twodimensional elliptic streamlines was seen in Thompson et al. (2001). Leweke \& Williamson (1998) suggested that when two counter-rotating vortices balance each other, the radial component of the strain field leads the disturbance to grow exponentially.

Lanzerstorfer \& Kuhlmann (2012) observed that the combination of the flow deceleration near the reattachment point, a lift up process on both sides of the bulk flow between the primary and the secondary recirculation bubbles, and an amplification due to streamline convergence near and in the separated flow regions is the cause of the flow instability in the flow over backward-facing step with expansion ratio of 0.5. The same observation was made by Wee et al. (2004) where they found 
(a) $\beta=0.2, R e=123$ and $k=2.8$

(i)

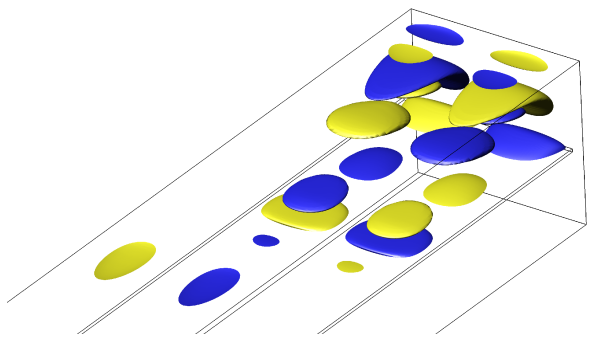

(b) $\beta=0.5, R e=278$ and $k=2$

(i)

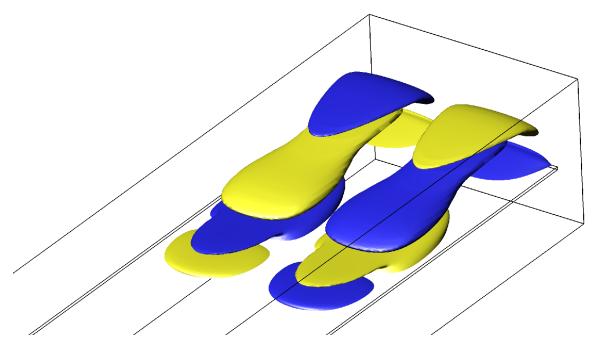

(c) $\beta=1, R e=397$ and $k=2$

(i)

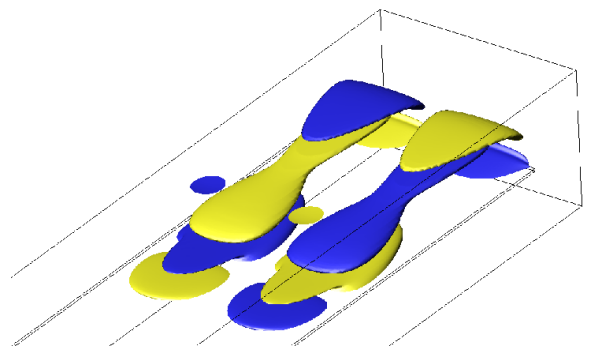

(d) $\beta=2, R e=380$ and $k=2$

(i)

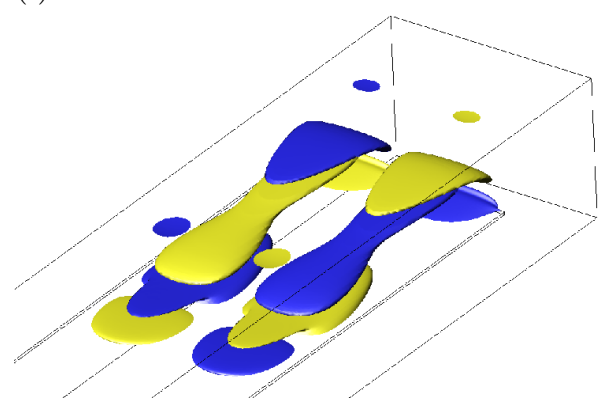

(ii)

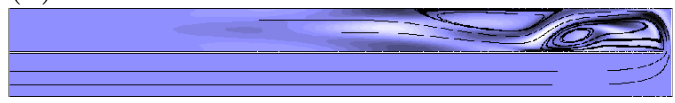

(iii)

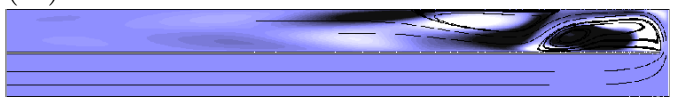

(ii)

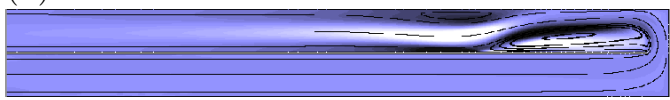

(iii)

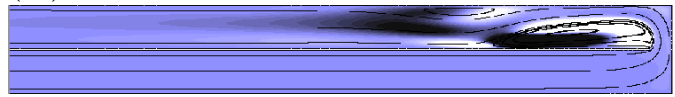

(ii)

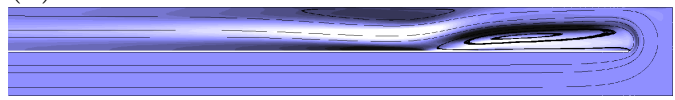

(iii)

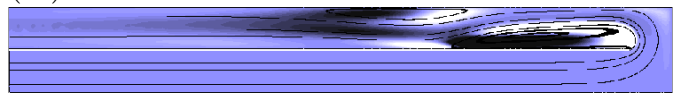

(ii)

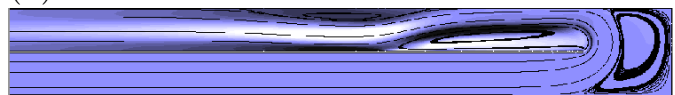

(iii)

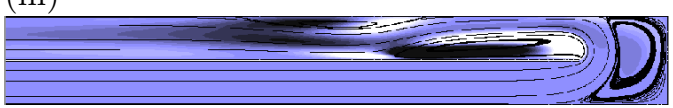

Figure 19: Visualisation of the real part of leading eigenmodes at (a) $\beta=0.2$, (b) $\beta=0.5$, (c) $\beta=1$ and (d) $\beta=2$, consisting of (i) a three-dimensional visualisation of the streamwise $(x$ component) of vorticity, (ii) spanwise ( $z$-component) vorticity and (iii) spanwise velocity contours overlaid with the base flow streamlines. Dark-mid-light shading denotes negative-zero-positive levels, respectively, while (ii) and (iii) are plotted on the plane $z=0$. 
that the backward-facing step flow was locally absolutely unstable near the middle of the primary recirculation bubble. The backflow was found to be high and the shear layer was sufficiently thick to support an absolutely unstable mode; hence, an absolute mode was more likely to originate in the middle of the bubble. By contrast, Marquillie \& Ehrenstein (2003) studied a flow behind a bump and observed that the structural changes near the reattachment point of the primary recirculation bubble behind the bump triggered an abrupt local transition from convective to absolute instability. It is observed that for the flow around a 180-degree sharp bend, as Re increases, the location of the peak backflow in the primary recirculation bubble shifted towards the reattachment point. The close gap between the peak backflow and the reattachment point means that the flow is strongly decelerated upon approaching the reattachment point. Interestingly, the peak backflow at the onset of instability in this study for $0.3 \lesssim \beta \lesssim 2$ is consistently located about $0.22 L_{R}$ from the reattachment point which is also the same location of the peak perturbation spanwise velocity from the linear stability analysis (figure 19 biii, ciii, diii). This suggests that as in the other geometries mentioned, the instability in the 180-degree sharp bend for $\beta \geq 0.3$ is localised near the peak of back flow intensity.

The oscillatory critical mode at $\beta=0.2$ exhibits strong spanwise velocities at the upstream end of the primary recirculation bubble and quite strong values around the intense vortex in the bubble. This is strongly consistent with a mechanism involving a centrifugal instability around the intense vortex. A similar mode was seen by Lanzerstorfer \& Kuhlmann (2012) in the flow over a backward-facing step with a very small opening. There, the perturbations were found to be a spanwise travelling wave that displaces the jet and the intense vortex periodically.

The structure of the mode that destabilizes the flow at $\beta=0.5, R e=278$ and $k=2$ is shown in figure 19 (b) in the isosurface plots of streamwise vorticity, spanwise vorticity and $w$ velocity contours. The isosurface consists of positive (light) and negative (dark) vorticity contours located almost entirely in the primary recirculation bubble, near the separation and reattachment points. The spanwise vorticity contour plot shows that there is a pair of counter-rotating vortices in the primary recirculation bubble which resemble the flow in a partially blocked channel (Griffith et al. 2007). The spanwise velocity contours are also qualitatively similar to those of the unstable mode in the flow over a backward-facing step (Barkley et al. 2002 ) where a "flat roll" (i.e. a roll in a horizontal plane about a vertical axis) mode structure exists in the bubble near the reattachment point. Across the opening ratios studied, the same mode structure has been found for all real primary leading eigenmodes indicated by the solid symbols in figure 18 .

The same type of plots describing the dominant eigenmodes for $\beta=0.2, R e=123$ and $k=2.8$ are shown in figure 19 (a). The mode appears to grow in the primary recirculation bubble near the separation point and upstream of the intense vortex close to the reattachment point. Both of these modes have strong $x$-component of perturbation vorticity in the upstream part of the primary recirculation bubble which is where Zhang \& Pothérat (2013) found secondary instability in their three-dimensional simulation of an unsteady flow at $R e=2000$ and $\beta=1$ with spanwise periodic domain of length 2 units (this is equivalent to the case of wavenumber $k=\pi$ in the present notations).

The structure of the most unstable eigenmode at very small wavenumber is shown in figure 20 as a plot of spanwise vorticity and $(u, v, w)$ velocity contours. The structure consist of spanwise vortices in the main bulk flow located between the primary and secondary recirculation bubbles. The spanwise velocity contour in figure 20(d) clearly shows that the bifurcating mode is located in the main bulk flow near the closed streamlines of both bubbles.

\subsection{Two-dimensional instability}

$\S 5.1$ describes how for $R e<R e_{\mathrm{c}}$ at any $\beta$, the flow is stable to two-dimensional infinitesimal perturbations $(k=0)$. The linear stability analysis found that all eigenvalues for $k=0$ are real 
(a) Streamwise vorticity

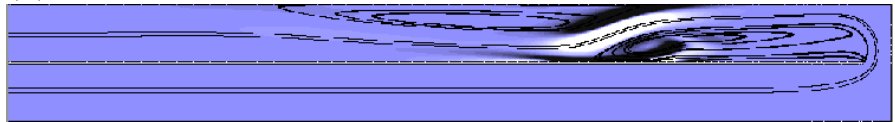

(b) $u$-velocity

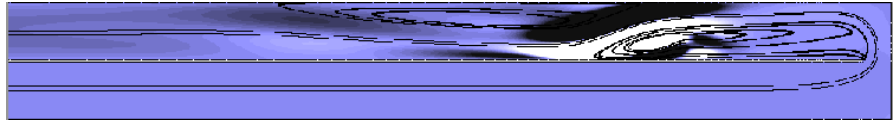

(c) $v$-velocity

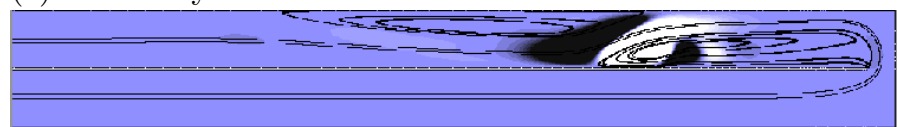

(d) $w$-velocity

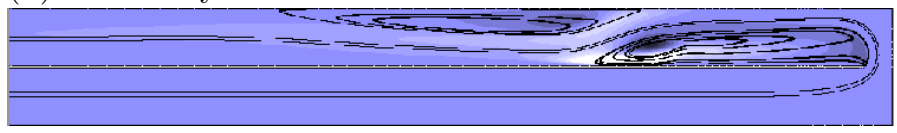

Figure 20: Structure of the real part of the leading eigenmode at $\beta=0.5, R e=400$ and $k=0.2$ depicted on the plane $z=0$ : flooded contours of (a) streamwise vorticity and (b) $u$, (c) $v$ and (d) $w$-velocity overlaid with the base flow streamlines. For clarity, only the vicinity of the bend is shown. Dark-mid-light shading denotes negative-zero-positive levels, respectively.

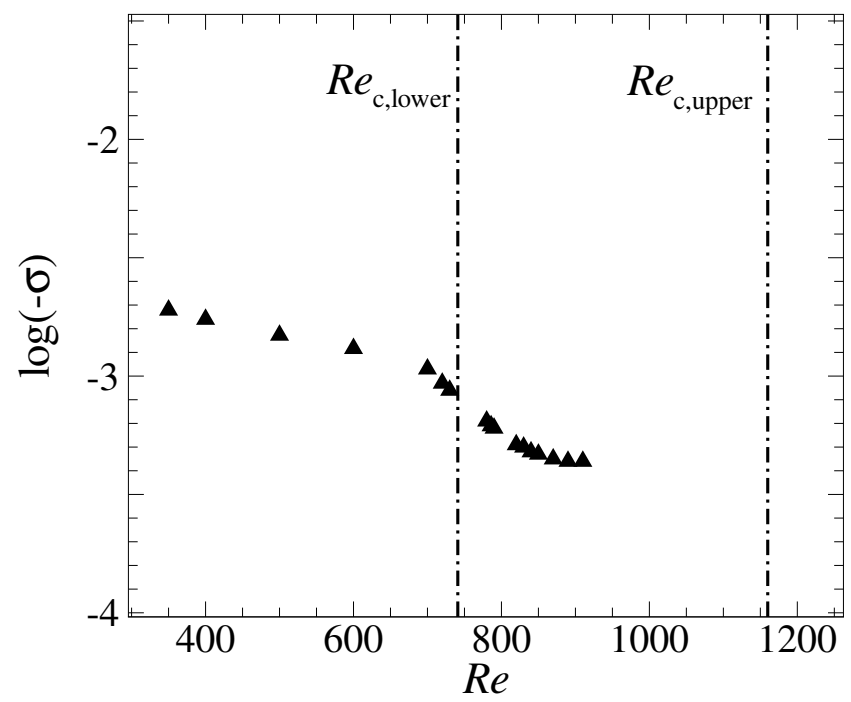

Figure 21: Leading two-dimensional $(k=0)$ eigenvalues for $\beta=1$. Due to the hysteresis, there are two $R e_{c}$ for this flow, $R e_{\mathrm{c} \text {,lower }}$ is the lowest $R e$ the flow can become unsteady, meanwhile $R e_{\mathrm{c} \text {, upper }}$ is the highest $R e$ the flow remains steady. The leading eigenvalues remain to have finite value of $\log (-\sigma)$ as $R e \rightarrow R e_{\mathrm{c}}$. Note that the flow will become linearly neutrally stable when $\sigma \rightarrow 0^{-}$, $\log (-\sigma) \rightarrow-\infty$ 
and have negative growth rate. The two-dimensional flow around a sharp 180-degree bend becomes unsteady at small Re depending on $\beta$, which is almost twice the critical value for the onset of threedimensional instability. From two-dimensional simulations, Zhang \& Pothérat (2013) observed that two-dimensional instability starts in the shear layer between the two steady recirculation bubbles and sheds throughout the whole width of the channel. This agrees with the mechanism found for the two-dimensional leading eigenmode. As $k \rightarrow 0$, a leading real eigenmode splits into two complex-conjugate pairs. Figure 21 is plotted similarly to figure 13 in Barkley et al. (2002); $\log (-\sigma)$ will approach $-\infty$ as $\sigma \rightarrow 0$ from below. However, the data here shows that the eigenmode growth rate remains negative as the critical Reynolds number is approached, lacking any evidence of a departure towards $-\infty$. While it was not possible due to compute time limitations to obtain stability data closer to $R e_{c \text {,upper }}$, this nevertheless suggests that the transition to unsteadiness of the steady-state two-dimensional solution branch is not due to a global linear instability. This is supported by our earlier observation that $R e_{c \text {,upper }}$ was resolution-sensitive. Similar observations to that shown in figure 21 were made for flow behind a backward-facing step (Barkley et al. 2002) and through a partially blocked channel (Griffith et al., 2007). The data exhibits a kink at $R e \approx 700$, were a sudden steepening in gradient is observed, before the data reverts to a nearly horizontal trend to higher Reynolds numbers. Barkley et al. (2002) observed a similar behaviour for the backward-facing step flow (in that case occurring at $R e \approx 1250$ ), and found by inspection of secondary eigenvalues that the kink occurred due to an "avoided crossing" of the two eigenmode branches.

\section{Non-linear analysis of the bifurcation to three-dimension- al state}

In this section, three-dimensional direct numerical simulation (DNS) is performed to assess the linear stability analysis predictions and to understand the nature of the bifurcation arising from the predicted linear instability modes. The three-dimensional algorithm exploits the spanwise homogeneity of the geometry, combining the two-dimensional spectral-element discretisation in the $x-y$ plane with a Fourier spectral method in the out-of-plane $z$-direction (for more details see Ryan et al., 2012, Sheard et al., 2009). The span of the domain in $z$ may be specified, and periodic boundary conditions are naturally enforced in the $z$-direction.

Tests were conducted to determine the dependence of computed three-dimensional solutions on the number of Fourier modes $N_{f}$ included in the simulations. In these tests, the spanwise wavenumber was selected to match a predicted linear instability mode above the critical Reynolds number, and a superposition of the two-dimensional base flow and three-dimensional eigenvector field of the predicted linear instability was used as an initial condition. The three-dimensional flow was then evolved in time until it saturated, at which point measurements of the domain integral of $|w|$ and a point-measurement of $w$-velocity were taken. Results are shown in table 5 , demonstrating that the solution having 8 Fourier modes is converged to within at least 2 and 3 significant figures to the result obtained with 16 modes. This is deemed sufficient to capture the non-linear growth behaviour and the saturated state of the mode, so 8 Fourier modes is employed hereafter.

\subsection{Non-linear evolution of the unstable modes}

Three-dimensional simulations were subsequently performed at selected $\beta$ and $R e$ combinations. The spanwise wavenumber in each case is deliberately set to match the corresponding linear instability eigenmode. It is acknowledged that this choice excludes long-wavelength features that may or may not arise. However, it facilitates an isolation of the instability mode under scrutiny. Figure 22 shows the time history of the spanwise velocity in these three-dimensional simulations 


$\begin{array}{ccccc}N_{f} & \int_{\Omega}|w| \mathrm{d} \Omega & \epsilon_{1} & \text { Point } w \text {-velocity } & \epsilon_{2} \\ 2 & 0.343542421 & 2.01 \% & -0.044052007 & 42.4 \% \\ 4 & 0.350601512 & 1.91 \% & -0.030932087 & 7.52 \% \\ 8 & 0.344016047 & 1.11 \% & -0.028768317 & 0.04 \% \\ 16 & 0.347868534 & - & -0.028780194 & -\end{array}$

Table 5: Convergence of the saturated three-dimensional DNS solution with number of Fourier modes included in the simulation $\left(N_{f}\right)$ for a test case having $R e=400, \beta=0.8$ and $k=2.0$. Percent differences between successive $\int_{\Omega}|w| \mathrm{d} \Omega$ and point $w$-velocity measurements are $\epsilon_{1}$ and $\epsilon_{2}$, respectively.

(a)

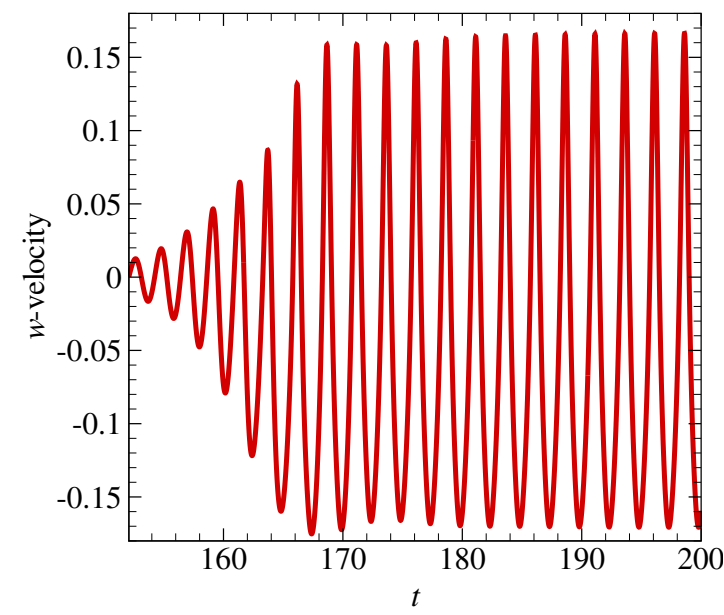

(b)

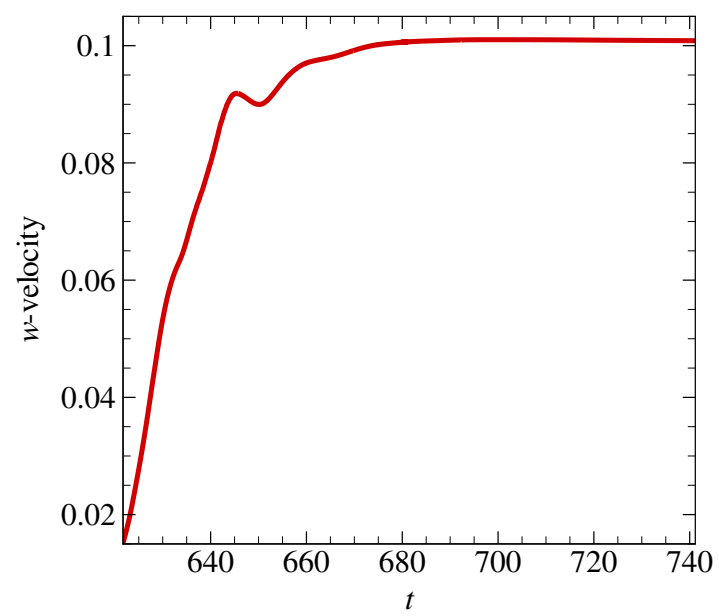

Figure 22: Time histories of $w$-velocity measured at a local point $(x, y, z)=(-2,0.52,1)$. Because $w$ is zero in the underlying two-dimensional base flow, non-zero $w$ is an indicator for three-dimensional flow development. In (a) $\beta=0.2, R e=160$ and the three-dimensional spanwise domain wavenumber is $k=4$. In (b) $\beta=1, R e=600$ and $k=4.5$.

$\begin{array}{cccccc}\beta & R e / R e_{3 \mathrm{D}} & k & \sigma(\mathrm{LSA}) & \sigma(3 \mathrm{D} \mathrm{DNS}) & \text { Percentage difference } \\ 0.2 & 1.12 & 3 & 0.06648 & 0.06667 & 0.27 \% \\ 0.4 & 1.16 & 3.2 & 0.09877 & 0.09909 & 0.3 \% \\ 0.5 & 1.22 & 2 & 0.03131 & 0.03133 & 0.011 \% \\ 0.8 & 1.03 & 2 & 0.01454 & 0.01455 & 0.043 \% \\ 2 & 1.55 & 4.5 & 0.20032 & 0.20102 & 0.35262 \%\end{array}$

Table 6: Comparison between the growth rates calculated from linear stability analysis (LSA) and three-dimensional DNS simulations. 
(a) $\beta=0.2, R e=160, k=4$

(i)

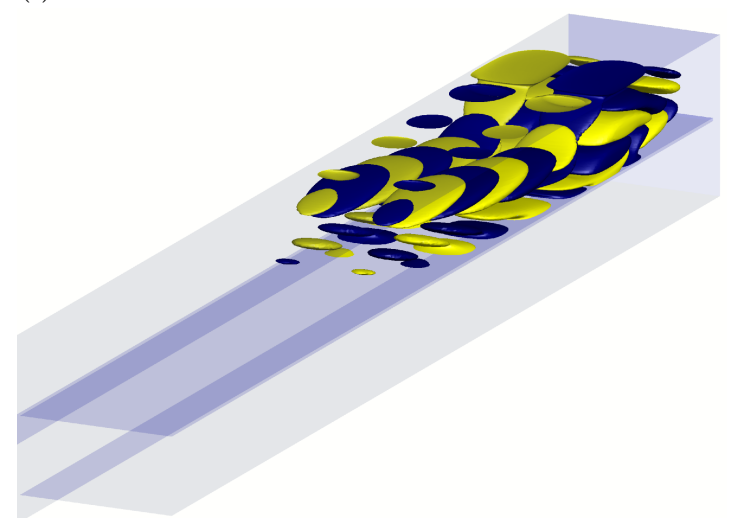

(b) $\beta=1, R e=600, k=4.5$

(i)

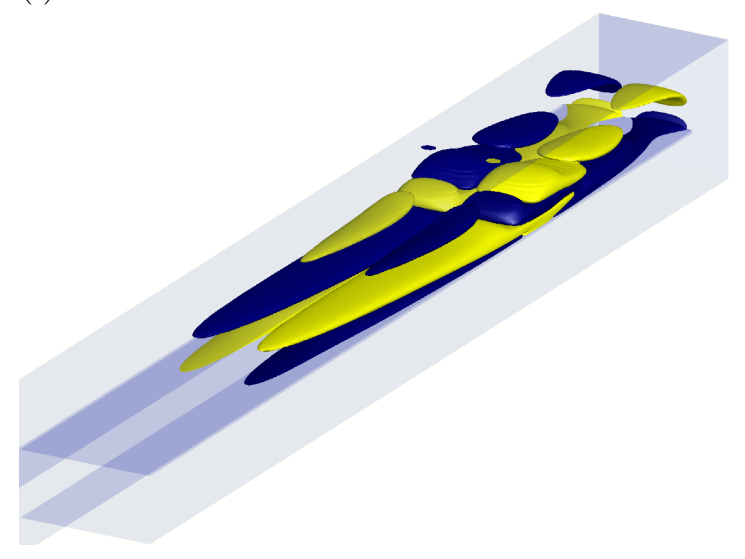

(ii)

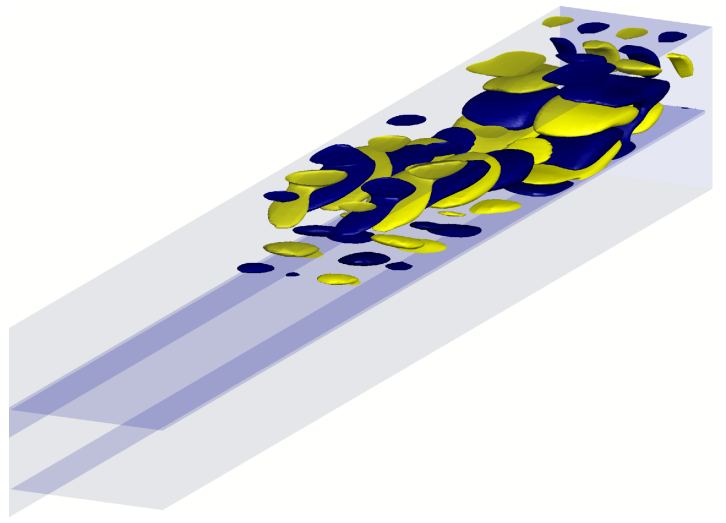

(ii)

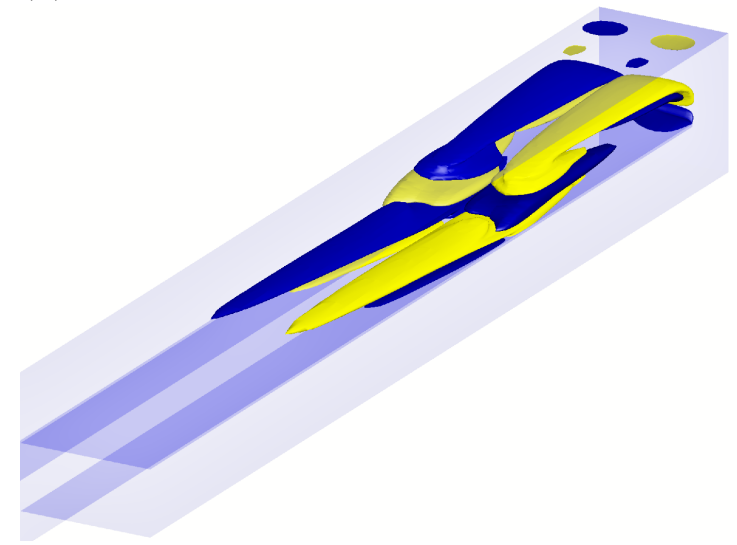

Figure 23: Visualisation of the three-dimensional disturbances via iso-surface plots of the (streamwise) $x$-component of vorticity for (a) $\beta=0.2, R e=160, k=4$ and (b) $\beta=1, R e=600, k=4.5$. (i) shows the leading eigenmode predicted by the linear stability analysis, and (ii) shows the saturated state of a three-dimensional DNS simulation. The saturated solution in (a ii) is oscillatory, and in (b ii) is steady-state. 
for (a) $\beta=0.2, R e=160, k=4$ and (b) $\beta=1, R e=600, k=4.5$. The oscillatory and the synchronous behavior in figure 22(a) and (b), respectively, agree well with the behavior of the leading eigenmodes predicted using linear stability analysis (ref. figure 12). From the time history of $w$-velocity, the growth rate of the perturbation can be calculated. Table 6 shows strong agreement between the growth rates of perturbation field obtained from the linear stability analysis and three-dimensional DNS simulation for five different cases. $\beta=2$ and $k=4.5$ is chosen to demonstrate the accuracy of the predictions on flow with fast growing perturbations. Figure 23 shows three-dimensional isosurface plots of streamwise vorticity for the same parameters as in figure 22, respectively, comparing the predicted three-dimensional eigenmode with the actual threedimensional state produced once the flow saturates following instability growth. The streamwise vorticity from the linear stability analysis (figure 23(a-b)(i)) has a strong resemblance to those of the three-dimensional DNS simulations (figure 23(a-b)(ii)). The strong agreement seen between the predicted eigenmode structure and the resulting saturated three-dimensional structure verifies that the linear stability analysis provides meaningful predictions of the three-dimensional nature of the flow. The Reynolds numbers in figure 23(a) and (b) are $28 \%$ and $51 \%$ higher than the critical Reynolds numbers for $\beta=0.2$ and 1, respectively. Both cases produce non-zero Fourier mode energy at saturation in the magnitude of $10^{-2}$ relative to the base flow energy, which is very small. The smaller the disturbance energy compared to the base flow energy, the closer the saturated state will be to the predicted infinitesimal eigenmode because the contribution of nonlinear terms is weaker.

From the three-dimensional DNS simulations, we found that the unsteady saturated state for $\beta=0.2$ persists to at least $R e=160$ three-dimensional simulations were not conducted beyond $R e=160$, though it might be anticipated based on the close agreement between the saturated threedimensional flow structure and the corresponding predicted linear instability mode, that a similar behaviour would extend to higher Reynolds numbers: linear stability analysis was performed up to $R e=200$, continuing to capture this mode. Meanwhile, for $\beta=0.5$, as Re increases, the saturated state changed from a steady at $R e=300$ and 400 to an unsteady saturated state at $R e=500$ with $k=4.5$. The same observation is predicted in figure 12 , demonstrating the applicability of linear stability analysis to this flow, and the rich tapestry of flow regimes across the $R e-\beta$ parameter space.

\subsection{Stuart-Landau model analysis}

In this subsection, analysis of the nonlinear features of the instability mode evolution is performed using a truncated Stuart-Landau equation. The Stuart-Landau model is valid in the vicinity of the transition Reynolds number, and has found wide application for classification of the non-linear characteristics of bifurcations in fluid flows. Examples include analysis of the Hopf bifurcation from steady-state flow past a circular cylinder producing the classical Kármán vortex street (Provansal et al., 1987; Duŝek et al., 1994; Schumm et al., 1994; Albarède \& Provansal, 1995; Thompson \& Le Gal , 2004), the regular (steady-to-steady) bifurcation breaking axisymmetry in the flow behind a sphere (Thompson et al., 2001 ), and three-dimensional transition behind a cylinder (Henderson \& Barkley, 1996; Sheard et al., 2003), staggered cylinders (Carmo et al. 2008), and rings (Sheard et al. | $|2004 b| a)$. The model describes the growth and saturation of perturbation as (Landau \& Lifshitz, 1976)

$$
\frac{\mathrm{d} A}{\mathrm{~d} t}=(\sigma+\mathrm{i} \omega) A-l(1+\mathrm{i} c)|A|^{2} A+\ldots,
$$

where $A$ is the complex amplitude of the evolving instability as a function of time, and the right side of the equation represents the first two terms of a series expansion. The growth rate and angular frequency of the mode in the linear regime $(|A| \rightarrow 0)$ are respectively denoted by $\sigma$ and $\omega$, while weakly non-linear properties are determined by the second term on the right hand side. The 
(a)

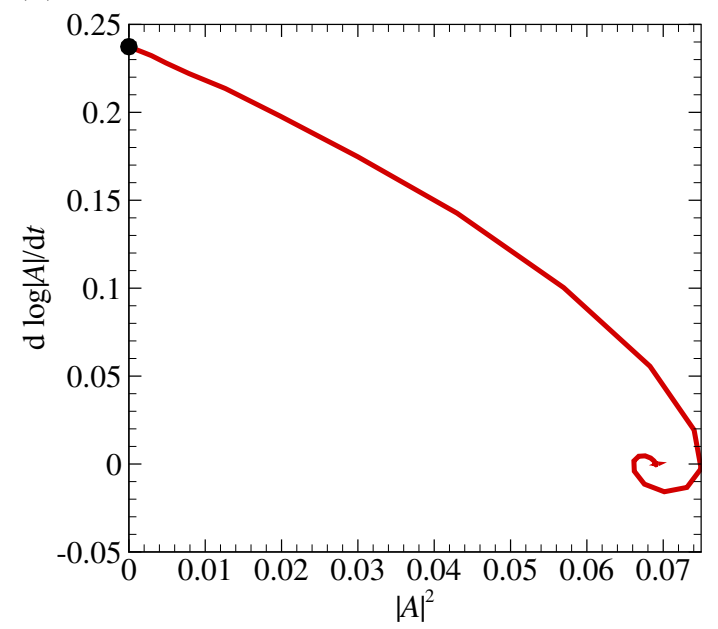

(b)

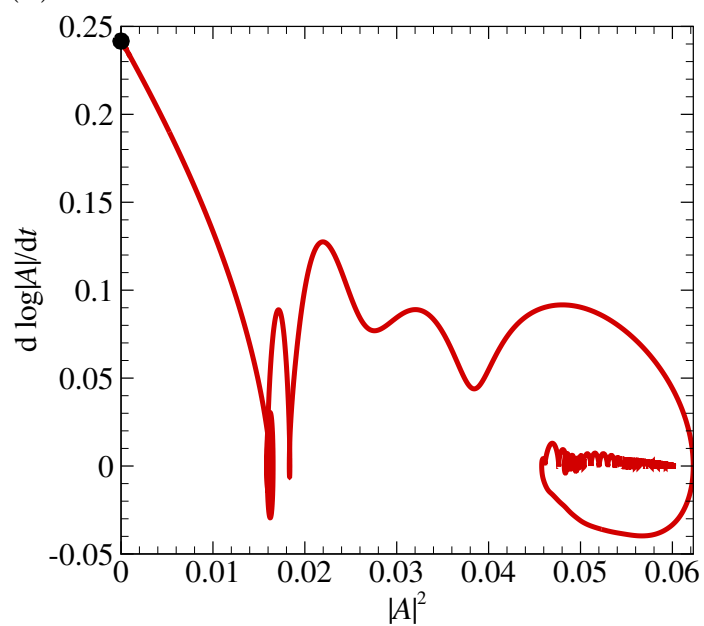

Figure 24: The time derivative of mode amplitude logarithm plotted against the square of the amplitude for (a) $\beta=0.2, R e=160, k=4$ and (b) $\beta=0.5, R e=400, k=3.5$ demonstrating supercritical behaviour. The solid circle symbol represents the linear stability analysis predicted growth rate.

sign of $l$ dictates whether the mode evolution is via a supercritical $(l>0)$ or subcritical $(l<0)$ bifurcation, and any frequency shift is described by Landau constant $c$ (Dusek et al., 1994; Le Gal et al., 2001, Thompson et al., 2001). A common treatment (Le Gal et al., 2001; Thompson et al., $2001)$ is to decompose $A(t)$ into magnitude and phase components as

$$
A(t)=\rho(t) \mathrm{e}^{i \phi(t)},
$$

where $\rho(t)=|A(t)|$ and $\phi(t)=\arg (A(t))$. Substitution of equation (16) into (15), separation into real and imaginary parts, and simplification yields separate equations for amplitude and phase, i.e.

$$
\begin{aligned}
& \frac{\mathrm{d} \rho}{\mathrm{d} t}=\sigma \rho-l \rho^{3}, \\
& \frac{\mathrm{d} \phi}{\mathrm{d} t}=\omega-l c \rho^{2} .
\end{aligned}
$$

It is convenient to then manipulate (17) as

$$
\frac{\mathrm{d}(\log \rho)}{\mathrm{d} t}=\frac{1}{\rho} \frac{\mathrm{d} \rho}{\mathrm{d} t}=\sigma-l \rho^{2} .
$$

Hence a positive slope $(-l)$ in a plot of $\mathrm{d}(\log |A|) / \mathrm{d} t$ against $|A|^{2}$ will indicate a subcritical bifurcation, while a negative slope corresponds to a supercritical bifurcation. Whether a supercritical bifurcation is of a pitchfork or Hopf type is dependent on whether the growing mode is synchronous or oscillatory.

The envelope of energy in the non-zero spanwise Fourier modes of the three-dimensional simulations was taken as a global amplitude measure $\left(|A|^{2}\right)$ of the growing three-dimensional instabilities. Figure 24 plots the time derivative of amplitude logarithm against the square of the amplitude for two different cases: (a) $\beta=0.2, R e=160, k=4$, which grows from an oscillatory mode, and (b) $\beta=0.5, R e=400, k=3.5$, which grows from a synchronous mode. The behaviour shown 
for $\beta=0.5$ is consistent with that found at larger $\beta$. The nearly linear variation with a negative gradient towards small $|A|^{2}$ shown in both plots indicates that the transition in both cases occurs through a supercritical bifurcation. This is consistent with other confined flow featuring recirculation bubbles such as the flow past a backward facing step (Kaiktsis et al. , 1991), through a sudden expansion in a circular pipe (Mullin et al. 2009 ), and past a sphere (Tomboulides \& Orszag, 2000). The oscillatory and synchronous behaviour of the modes in (a) and (b) reveal them to occur through supercritical Hopf and pitchfork bifurcations, respectively.

In addition to the aforementioned sub- and supercritical bifurcation scenarios, another possibility is that of a transcritical bifurcation. A dynamical system producing transcritical behaviour takes the form

$$
\frac{\mathrm{d} \rho}{\mathrm{d} t}=\sigma \rho-l \rho^{2},
$$

which differs from the amplitude part of the Stuart-Landau model 17 by the replacement of $\rho^{3}$ with $\rho^{2}$ in the non-linear term on the right hand side. Under an analogous manipulation,

$$
\frac{\mathrm{d}(\log \rho)}{\mathrm{d} t}=\sigma-l \rho=\sigma-l \sqrt{\rho^{2}} .
$$

The $\sqrt{\rho^{2}}$ term indicates that an infinite gradient would present at $|A|^{2}=0$ in a plot of $\mathrm{d}(\log |A|) / \mathrm{d} t$ against $|A|^{2}$. The absence of such a behaviour in figure 24 rules out a transcritical bifurcation. Similarly, a transcritical dynamical system expressed in terms of the complex amplitude $A$,

$$
\frac{\mathrm{d} A}{\mathrm{~d} t}=(\sigma+i \omega) A-l(1+i c) A^{2},
$$

features a real component that simplifies to

$$
\frac{\mathrm{d}(\log \rho)}{\mathrm{d} t}=\sigma-l \sqrt{\rho^{2}}[\cos \phi-c \sin \phi] .
$$

In the limit $|A|=\rho \rightarrow 0$, the oscillation described by the trigonometric term produces gradients in $\mathrm{d}(\log |A|) / \mathrm{d} t$ as a function of $|A|^{2}$ that approach infinity; the absence of this behaviour in figure 24 again supports the present classification of these bifurcations as being supercritical.

\section{Conclusions}

We conducted a linear stability analysis to characterise the onset of unsteadiness in the flow around a 180-degree sharp bend. We considered a range of opening ratios $\beta$ spanning all regimes from a jet-like flow through a small aperture to flow topologies involving a recirculation within the turning part (Zhang \& Pothérat, 2013). In all cases, we found the base flow with steady bubble in the outlet to be unstable to infinitesimal perturbations at a finite critical Reynolds number $R e_{\mathrm{c}}$, that increases monotonically with the effective opening ratio $\beta_{\text {eff }}$. $\beta_{\text {eff }}$ measures the actual width of the main stream in the turning part. Consequently $R e_{\mathrm{c}}$ increases monotonically with the geometric opening ratio $\beta$ so long as the mean stream occupies the whole turning part (up to $\beta \sim 1$ ). By contrast, for high opening ratios, $\operatorname{Re}_{c}(\beta)$ decreases asymptotically to $\operatorname{Re}_{c}\left(\beta_{\mathrm{eff}} \simeq 0.8\right)$, because the recirculation in the turning part reduce the effective width available to the main stream.

The linear stability analysis revealed three types of leading eigenmodes. In the subcritical range, the leading eigenmode is reminiscent of long-wave Taylor-Görtler vortices localised in the main stream between the two recirculation bubbles attached to either outlet walls. This mode was, however, never found to become unstable. As Re was increased, two unstable branches emerged that were respectively associated a real eigenvalue and a complex-conjugate pair of eigenvalues. 
The former dominates for $\beta \geq 0.3$. The corresponding perturbation has a spanwise wavenumber $k \simeq 2$ and is confined within the first recirculation region, with maximum intensity where the back flow is most intense. For $\beta=0.2$, by contrast unsteadiness sets in via the second branch under the form of a spanwise oscillating mode, akin to that found in backward facing step flows with small opening ratios (Lanzerstorfer \& Kuhlmann, 2012).

In all cases, critical modes were three-dimensional. Accordingly, locally unstable two-dimensional modes (i.e. having zero spanwise wavenumber) are only found at higher Reynolds numbers than three-dimensional modes, but do not grow through a global instability. They drive a KelvinHelmholtz instability in the main stream between the two recirculating bubbles that is consistent with the DNS of Zhang \& Pothérat (2013).

Analysis of the non-linear evolution of dominant instability modes using three-dimensional DNS and the Stuart-Landau equation demonstrated that transition from two-dimensional to three-dimensional flow consistently occurred through a supercritical bifurcation: a supercritical Hopf bifurcation at small $\beta$ and a supercritical pitchfork bifurcation at large $\beta$.

A. M. S. is supported by the Ministry of Education Malaysia and International Islamic University Malaysia. This research was supported by Discovery Grants DP120100153 and DP150102920 from the Australian Research Council, and was undertaken with the assistance of resources from the National Computational Infrastructure (NCI), which is supported by the Australian Government. A. P. acknowledges support from the Royal Society under the Wolfson Research Merit Award Scheme (grant WM140032).

\section{References}

ABU-NADA, E. 2008 Application of nanofluids for heat transfer enhancement of separated flows encountered in a backward facing step. International Journal of Heat and Fluid Flow 29 (1), $242-249$.

Alam, M. \& Sandham, N. D. 2000 Direct numerical simulation of 'short' laminar separation bubbles with turbulent reattachment. J. Fluid Mech. 403, 223-250.

Albarède, P. \& Provansal, M. 1995 Quasi-periodic cylinder wakes and the Ginzburg-Landau equation. J. Fluid Mech. 291, 191-222.

Armaly, B. F., Durst, F., Pereira, J. C. F. \& Schonung, B. 1983 Experimental and theoretical investigation of backward-facing step flow. J. Fluid Mech 127 (473), 20.

Astarita, T. \& CARdone, G. 2000 Thermofluidynamic analysis of the flow in a sharp $180^{\circ}$ turn channel. Exp. Therm. Fluid Sci. 20 (3-4), 188-200.

Barkley, D., Blackburn, H. M. \& Sherwin, S. J 2008 Direct optimal growth analysis for timesteppers. Int. J. Numer. Methods Fluids 57 (9), 1435-1458.

Barkley, D., Gomes, M. G. M \& Henderson, R. D. 2002 Three-dimensional instability in flow over a backward-facing step. J. Fluid Mech. 473, 167-190.

Barkley, D. \& Henderson, R. D. 1996 Three-dimensional Floquet stability analysis of the wake of a circular cylinder. J. Fluid Mech. 322, 215-242.

Barleon, L., Casal, V. \& Lenhart, L. 1991 MHD flow in liquid-metal-cooled blankets. Fusion Engineering and Design 14 (3), 401-412.

Barleon, L., Mack, K. J. \& Stieglitz, R. 1996 The MEKKA-facility: A Flexible Tool to Investigate MHD-flow Phenomena. Forschungszentrum Karlsruhe.

BARTON, I. E. 1997 The entrance effect of laminar flow over a backward-facing step geometry. International Journal for Numerical Methods in Fluids 25 (6), 633-644.

Blackburn, H. M., Barkley, D. \& Sherwin, S. J. 2008 Convective instability and transient growth in flow over a backward-facing step. J. Fluid Mech. 603, 271-304. 
Boccaccini, L. V., Giancarli, L., Janeschitz, G., Hermsmeyer, S., Poitevin, Y., Cardella, A. \& Diegele, E. 2004 Materials and design of the European DEMO blankets. $J$. Nucl. Mater. 329, 148-155.

Brede, M., Eckelmann, H. \& Rockwell, D. 1996 On secondary vortices in the cylinder wake. Phys. Fluids 8 (8), 2117-2124.

BüHLER, L. 2007 Liquid metal magnetohydrodynamics for fusion blankets. Magnetohydrodynamics pp. $171-194$.

Carmo, B. S., Sherwin, S. J., Bearman, P. W. \& Willden, R. H. J. 2008 Wake transition in the ow around two circular cylinders in staggered arrangements. J. Fluid Mech. 597.

Chung, Y. M., Tucker, P. G. \& Roychowdhury, D. G. 2003 Unsteady laminar flow and convective heat transfer in a sharp $180^{\circ}$ bend. Int. J. Heat Fluid Flow 24 (1), 67-76.

Cruchaga, M. A. 1998 A study of the backward-facing step problem using a generalized streamline formulation. Communications in Numerical Methods in Engineering 14 (8), 697-708.

Drazin, P. G. \& ReID, W. H. 2004 Hydrodynamic stability. Cambridge university press.

DuŝeK, J., Le Gal, P. \& Fraunié, P. 1994 A numerical and theoretical study of the first Hopf bifurcation in a cylinder wake. J. Fluid Mech. 264, 59-80.

ERTURK, E. 2008 Numerical solutions of 2-d steady incompressible flow over a backward-facing step, part i: High Reynolds number solutions. Computers \& Fluids 37 (6), 633-655.

Ghia, K. N., Osswald, G. A. \& GhiA, U. 1989 Analysis of incompressible massively separated viscous flows using unsteady Navier-Stokes equations. Int. J. Numer. Methods Fluids 9 (8), 1025-1050.

Griffith, M. D., Leweke, T., Thompson, M. C., Hourigan, K. et al. 2008 Steady inlet flow in stenotic geometries: convective and absolute instabilities. J. Fluid Mech.. 616, 111.

Griffith, Martin D, Thompson, Mark C, Leweke, T, Hourigan, K \& Anderson, WarWICK P 2007 Wake behaviour and instability of flow through a partially blocked channel. J. Fluid Mech.. 582 (1), 319-340.

Hammond, D. A. \& Redekopp, L. G. 1998 Local and global instability properties of separation bubbles. European Journal of Mechanics-B/Fluids 17 (2), 145-164.

Henderson, RonAld D 1997 Nonlinear dynamics and pattern formation in turbulent wake transition. J. Fluid Mech. 352, 65-112.

Henderson, R. D. \& BARKLey, D. 1996 Secondary instability in the wake of a circular cylinder. Phys. Fluids 8, 1683.

Hirota, M., Fujita, H., Syuhada, A., Araki, S., Yoshida, T. \& Tanaka, T. 1999 Heat/mass transfer characteristics in two-pass smooth channels with a sharp 180-deg turn. Int. J. Heat Mass Transfer 42 (20), 3757-3770.

Hussam, W. K., Thompson, M. C. \& Sheard, G. J. $2012 a$ Enhancing heat transfer in a high Hartmann number magnetohydrodynamic channel flow via torsional oscillation of a cylindrical obstacle. Phys. Fluids 24 (11), 113601.

Hussam, W. K., Thompson, M. C. \& Sheard, G. J. $2012 b$ Optimal transient disturbances behind a circular cylinder in a quasi-two-dimensional magnetohydrodynamic duct flow. Phys. Fluids 24 (2), 024105.

Johnson, T. A. \& Patel, V. C. 1999 Flow past a sphere up to a Reynolds number of $300 . J$. Fluid Mech. 378, 19-70.

Kaiktsis, Lambros, Karniadakis, George Em \& Orszag, Steven A 1991 Onset of threedimensionality, equilibria, and early transition in flow over a backward-facing step. J. Fluid Mech. 231, 501-528.

Karniadakis, G. E., Israeli, M. \& Orszag, S. A. 1991 High-order splitting methods for the incompressible Navier-Stokes equations. J. Comput. Phys 97 (2), 414-443.

Kirillov, I. R., Reed, C. B., Barleon, L. \& Miyazaki, K. 1995 Present understanding of 
MHD and heat transfer phenomena for liquid metal blankets. Fusion Eng. Des. 27, 553-569.

Krall, K. M. \& Sparrow, E. M. 1966 Turbulent heat transfer in the separated, reattached, and redevelopment regions of a circular tube. J. Heat Transfer 88 (1), 131-136.

Landau, LD \& Lifshitz, EM 1976 Mechanics pergamon press. New York p. 93.

Lanzerstorfer, D. \& Kuhlmann, H. C. 2012 Global stability of the two-dimensional flow over a backward-facing step. J. Fluid Mech. 693, 1-27.

Larson, H. K. 1959 Heat transfer in separated flows. J. Aerospace Sci. 26 (11), 731-738.

Le Gal, Patrice, Nadim, Ali \& Thompson, Mark 2001 Hysteresis in the forced stuart-landau equation: application to vortex shedding from an oscillating cylinder. J. Fluids Struct. 15 (3), $445-457$.

Lehoucq, R. B., Sorenson, D. C. \& Yang, C. 1998 ARPACK Users' Guide: Solution of Large-Scale Eigenvalue Problems with Implicitly Restarted Arnoldi Methods. SIAM.

Leweke, T. \& Williamson, C. H. K. 1998 Cooperative elliptic instability of a vortex pair. J. Fluid Mech. 360, 85-119.

Liou, T-M, Chen, C-C, Tzeng, Y-Y \& Tsai, T-W 2000 Non-intrusive measurements of nearwall fluid flow and surface heat transfer in a serpentine passage. Int. J. Heat Mass Transfer 43 (17), 3233-3244.

Liou, T-M, Tzeng, Y-Y \& Chen, C-C 1999 Fluid flow in a 180 deg sharp turning duct with different divider thicknesses. J. Turbomach. 121 (3), 569-576.

Marquillie, M. \& Ehrenstein, U. W. E. 2003 On the onset of nonlinear oscillations in a separating boundary-layer flow. J. Fluid Mech. 490, 169-188.

Metzger, D. E. \& Sahm, M. K. 1986 Heat transfer around sharp 180-deg turns in smooth rectangular channels. J. Heat Transfer 108 (3), 500-506.

Moffatt, H. K. 1985 Magnetostatic equilibria and analogous euler flows of arbitrarily complex topology. part 1. fundamentals. J. Fluid Mech. 159, 359-378.

Mullin, T, Seddon, JRT, Mantle, MD \& Sederman, AJ 2009 Bifurcation phenomena in the flow through a sudden expansion in a circular pipe. Physics of Fluids (1994-present) 21 (1), 014110 .

Natarajan, R. \& Acrivos, A. 1993 The instability of the steady flow past spheres and disks. J. Fluid Mech. 254, 323-344.

Neild, A., Ng, T. W., Sheard, G. J., Powers, M. \& Oberti, S. 2010 Swirl mixing at microfluidic junctions due to low frequency side channel fluidic perturbations. Sensors and Actuators B: Chemical 150 (2), 811-818.

Provansal, M., Mathis, C. \& Boyer, L. 1987 Bénard-von Kármán instability: Transient and forced regimes. J. Fluid Mech. 182, 1-22.

Ryan, K., Butler, C. J. \& Sheard, G. J. 2012 Stability characteristics of a counter-rotating unequal strength Batchelor vortex pair. J. Fluid Mech. 696, 374-401.

Schumm, M., Berger, E. \& Monkewitz, P. 1994 Self-excited oscillations in the wake of twotimensional bluff bodies and their control. J. Fluid Mech. 271, 17-53.

ShEARD, G. J. 2011 Wake stability features behind a square cylinder: focus on small incidence angles. Journal of Fluid Structure 27 (5), 734-742.

Sheard, G. J., Fitzgerald, M. J. \& RYan, K. 2009 Cylinders with square cross-section: wake instabilities with incidence angle variation. J. Fluid Mech. 630, 43-69.

Sheard, G. J., Thompson, M. C. \& Hourigan, K. 2003 A coupled Landau model describing the Strouhal-Reynolds number profile of a three-dimensional circular cylinder wake. Physics of Fluids (1994-present) 15 (9), L68-L71.

Sheard, G. J., Thompson, M. C. \& Hourigan, K. 2004a Asymmetric structure and non-linear transition behaviour of the wakes of toroidal bodies. Euro. J. Mech. B-Fluids 23 (1), 167-179.

Sheard, G. J., Thompson, M. C. \& Hourigan, K. $2004 b$ From spheres to circular cylinders: 
Non-axisymmetric transitions in the flow past rings. J. Fluid Mech. 506, 45-78.

TANEDA, S. 1979 Visualization of separating stokes flows. Journal of Physical Society of Japan 46, 1935-1942.

Thompson, M. C., Hourigan, K. \& Sheridan, J. 1996 Three-dimensional instabilities in the wake of a circular cylinder. Experimental Thermal and Fluid Science 12 (2), 190-196.

Thompson, Mark C. \& Le Gal, Patrice 2004 The Stuart-Landau model applied to wake transition revisited. European Journal of Mechanics-B/Fluids 23 (1), 219-228.

Thompson, M. C., Leweke, T. \& Williamson, C. H. K. 2001 The physical mechanism of transition in bluff body wakes. Journal of Fluids and Structures 15 (3), 607-616.

Tomboulides, A. G. \& Orszag, S. A. 2000 Numerical investigation of transitional and weak turbulent flow past a sphere. J. Fluid Mech. 416, 45-73.

Vo, T., Montabone, L. \& Sheard, G. J. 2014 Linear stability analysis of a shear layer induced by differential coaxial rotation within a cylindrical enclosure. J. Fluid Mech. 738, 299-334.

Vo, T., Montabone, L. \& Sheard, G. J. 2015 Effect of enclosure height on the structure and stability of shear layers induced by differential rotation. J. Fluid Mech. 765, 45-81.

WANG, T-S \& ChyU, M. K. 1994 Heat convection in a 180-deg turning duct with different turn configurations. J. Thermophys. Heat Transfer 8 (3), 595-601.

Wee, D., Yi, T., Annaswamy, A. \& Ghoniem, A. F. 2004 Self-sustained oscillations and vortex shedding in backward-facing step flows: Simulation and linear instability analysis. Phys. Fluids 16 (9), 3361-3373.

Williamson, C. H. K. 1988 Defining a universal and continuous Strouhal-Reynolds number relationship for the laminar vortex shedding of a circular cylinder. Phys. Fluids 31 (10), 27422744.

Zhang, L. \& Pothérat, A. 2013 Influence of the geometry on the two-and three-dimensional dynamics of the flow in a $180^{\circ}$ sharp bend. Phys. Fluids 25, 053605. 